\title{
A class of new exact solutions of the equations governing the steady plane flows of incompressible fluid of variable viscosity
}

\author{
Rana Khalid Naeem*, Mushtaq Ahmed \\ Department of Mathematics, University of Karachi Karachi-75270, Pakistan \\ *Corresponding author E-mail:2rknaeem@gmail.com
}

Copyright $\odot 2015$ Rana Khalid Naeem, Mushtaq Ahmed. This is an open access article distributed under the Creative Commons Attribution License, which permits unrestricted use, distribution, and reproduction in any medium, provided the original work is properly cited.

\begin{abstract}
The objective of this paper is to indicate a class of new exact solutions of the equations governing the steady plane flows of incompressible fluid of variable viscosity. The class consists of the stream function characterized by equation (2). Exact solutions are determined for $g(r)=$ const. and $g(r) \neq$ const. When $f(r)$ is arbitrary we can construct an infinite set of streamlines and the velocity components, viscosity function, generalized energy function $L$ and temperature distribution $T$. Therefore, an infinite set of solutions to flow equations. When $f(r)$ is not arbitrary, there are two values of $f(r)$ and therefore, two exact solutions to flow equations. The streamlines are presented through Fig.(1-56) for some chosen from of $f(r)$.

Keywords: A Class of Exact Solutions; Exact Solutions to the Flow Equations of Incompressible; Variable Viscosity; Navier-Stokes Equations; New Exact Solutions Variable Viscosity.
\end{abstract}

\section{Introduction}

Due to complex mathematical structure of the fluid flow equations, it is extremely difficult to achieve exact solutions. However, researchers have developed methods/techniques through which some exact solutions were determinable. The readers interested in these methods/techniques may refer to [1]-[23] and the references therein.

The aim of this paper is to indicate a class of new exact solutions of the equations describing the steady plane flows of incompressible fluid of variable viscosity. The aim is achieved by transforming flows equations into Martin system $(\phi, \psi)$. In Martin system, the coordinate lines $\psi=$ constant represents streamlines and coordinate lines $\phi=$ constant are left arbitrary. We take $\phi=r(x, y)$ to achieve our aim. When $f(r)$ is arbitrary an infinite set of velocity components implying an infinite set of solutions to flow equation. When $f(r)$ is not arbitrary there are only two values of $f(r)$ indicating a set of two solutions.

The streamlines of the class of flows under consideration are characterized by

$$
\frac{\theta-f(r)}{g(r)}=\text { const }
$$

where $f(r)$ and $g(r) \neq 0$ are continuously differentiable functions and $r, \theta$ the polar coordinates. The equation, with loss of generality, implies

$$
\theta=f(r)+g(r) v(\psi)
$$

where $v(\psi)$ is unknown function such that $v^{\prime}(\psi) \neq 0$. 
The paper is organized as follow: In section (2), we give basic flow equations and transform them into Martin system. In section (3), we take $\phi=r(x, y)$ and transform the equations of section (2) in to a new system of equations. The solutions to new system of equations are determined. In section (4) we discuss the solutions of section (3). In section (5) we present conclusions.

\section{Basic flow equations}

The basic non-dimensional equations of motion governing a steady plane flow of an incompressible fluid of variable viscosity, in the absence of external force with no heat addition are

$u_{x}+v_{y}=0$

$u u_{x}+v u_{y}=-p_{x}+\frac{1}{\mathrm{R}_{\mathrm{e}}}\left[\left(2 \mu u_{x}\right)_{x}+\left\{\mu\left(u_{y}+v_{x}\right)\right\}_{y}\right]$

$u v_{x}+v v_{y}=-p_{y}+\frac{1}{\mathrm{R}_{\mathrm{e}}}\left[\left(2 \mu v_{y}\right)_{y}+\left\{\mu\left(u_{y}+v_{x}\right)\right\}_{x}\right]$

$u T_{x}+v T_{y}=\frac{T_{x x}+T_{y y}}{\mathrm{R}_{\mathrm{e}} \mathrm{P}_{\mathrm{r}}}+\frac{\mathrm{E}_{\mathrm{c}}}{\mathrm{R}_{\mathrm{e}}}\left[2 \mu\left(u_{x}^{2}+v_{y}{ }^{2}\right)+\mu\left(u_{y}+v_{x}\right)^{2}\right]$

where $p$ is the pressure, $\mu$ is the viscosity, $T$ is the temperature, $u$ and $v$ are velocity components.

On introducing the vorticity function $w$, the total energy function $L$, the function $A$ and $B$ defined by

$$
w=v_{x}-u_{y}
$$

$L=p+\frac{1}{2}\left(u^{2}+v^{2}\right)-\frac{2 \mu u_{x}}{\mathrm{R}_{\mathrm{e}}}$

$A=\mu\left(u_{y}+v_{x}\right), \quad B=4 \mu u_{x}$

The system of equations $(3-6)$ can be rewritten as

$u_{x}+v_{y}=0$

$-v w=-L_{x}+\frac{A_{y}}{\mathrm{R}_{\mathrm{e}}}$

$u w=-L_{y}-\frac{B y}{\mathrm{R}_{\mathrm{e}}}+\frac{A_{x}}{\mathrm{R}_{\mathrm{e}}}$

$u T_{x}+v T_{y}=\frac{T_{x x}+T_{y y}}{\mathrm{R}_{\mathrm{e}} \mathrm{P}_{\mathrm{r}}}+\frac{\mathrm{E}_{\mathrm{c}}}{4 \mu \mathrm{R}_{\mathrm{e}}}\left(B^{2}+4 A^{2}\right)$

Now following Martin we introduce the curvilinear coordinate $(\phi, \psi)$ in the physical plane in which the coordinate lines $\psi=$ constant are the streamlines and the coordinate lines $\phi=$ constant left arbitrary.

In transforming the flow equations into curvilinear coordinates $(\phi, \psi)$, Martin considered the transformation defined by

$x=x(\phi, \psi)$ and $y=y(\phi, \psi)$

The transformation in (14) defines a system of curvilinear coordinates $(\phi, \psi)$ in the physical plane $(x, y)$ such that the Jacobian, $J=\frac{\partial(x, y)}{\partial(\phi, \psi)}$ of the transformation is non-zero and finite. The first fundamental form $d s^{2}$ in $(\phi, \psi)$ system is given by

$d s^{2}=E(\phi, \psi) d \phi^{2}+2 F(\phi, \psi) d \phi d \psi+G(\phi, \psi) d \psi^{2}$ 
where

$E(\phi, \psi)=x_{\phi}^{2}+y_{\phi}^{2}, F(\phi, \psi)=x_{\phi} x_{\psi}+y_{\phi} y_{\psi}, G(\phi, \psi)=x_{\psi}^{2}+y_{\psi}^{2}$

Differentiating equation (14) with respect to $x$ and $y$, and solving the resulting equations for $\psi_{x}, \psi_{y}, \phi_{x}, \phi_{y}$ yield:

$x_{\phi}=J \psi_{y}, \quad x_{\psi}=-J \phi_{y}, \quad y_{\phi}=-J \psi_{x}, \quad y_{\psi}=J \phi_{x}$

wherein

$J= \pm \sqrt{E G-F^{2}}= \pm\left(x_{\phi} y_{\psi}-y_{\varphi} x_{\psi}\right)= \pm W$

If $\alpha$ is the angle between the tangent at the point $P(x, y)$ to the coordinate line $\psi=$ constant and the $\mathrm{x}$-axis, then

$\tan \alpha=\frac{y_{\phi}}{x_{\phi}}$

Equation(17), on utilizing equation (19), gives

$$
\begin{aligned}
& x_{\phi}=\sqrt{E} \operatorname{Cos} \alpha, x_{\psi}=\frac{1}{\sqrt{E}}[F \operatorname{Cos} \alpha-J \operatorname{Sin} \alpha] \\
& y_{\phi}=\sqrt{E} \operatorname{Sin} \alpha, \quad y_{\psi}=\frac{1}{\sqrt{E}}\left[\begin{array}{l}
F \operatorname{Sin} \alpha+J \operatorname{Cos} \alpha]
\end{array}\right.
\end{aligned}
$$

The integrablity conditions

$x_{\psi \phi}=x_{\phi \psi} \quad y_{\psi \phi}=y_{\phi \psi}$

For $x$ and $y$, yield

$\alpha_{\phi}=\frac{J \Gamma_{11}^{2}}{E}, \alpha_{\psi}=\frac{J \Gamma_{12}^{2}}{E}$

wherein

$$
\Gamma_{11}^{2}=\frac{1}{2 W^{2}}\left[-F E_{\phi}+2 E F_{\phi}-E E_{\psi}\right], \Gamma_{12}^{2}=\frac{1}{2 W^{2}}\left[E G_{\phi}-F E_{\psi}\right]
$$

Equation (19), applying the integrability condition $\alpha_{\phi \psi}=\alpha_{\psi \phi}$ for $\alpha(\phi, \psi)$, yields

$K=\frac{1}{W}\left[\left(\frac{W \Gamma_{11}^{2}}{E}\right)_{\psi}-\left(\frac{W \Gamma_{12}^{2}}{E}\right)_{\phi}\right]$

where $\mathrm{K}$ is called the Gaussian curvature and equation (24) is called Gaussian equation. This equation represents a necessary condition that $E(\phi, \psi), F(\phi, \psi)$ and $G(\phi, \psi)$ are coefficients of the first fundamental form in equation (15). The system of equations $(10-13)$, on utilizing equations $(20-24)$, is transformed into a new system of equations given in the following theorem.

\section{Theorem I:}

If the streamlines $\psi=$ constant and the curves $\phi=$ constant left arbitrary, generate a curvilinear net in the physical plane, the equations $(10-13)$, are transformed in to the following system of equations

$q=\frac{\sqrt{E}}{W}$ 


$$
\begin{aligned}
-R_{e} w J E=R_{e} J E & \left.L_{\psi}+A_{\phi}\left(\left(F^{2}-J^{2}\right) \cos 2 \alpha-2 F J \sin 2 \alpha\right)+E A_{\psi}(J \sin 2 \alpha-F \cos 2 \alpha)\right)-B_{\phi}\left(\frac{1}{2}\left(F^{2}-J^{2}\right) \sin 2 \alpha+F J \cos 2 \alpha\right) \\
& +E B_{\psi}\left(\frac{1}{2} F \sin 2 \alpha+J \cos ^{2} \alpha\right)
\end{aligned}
$$

$0=-R_{e} J L_{\phi}+E A_{\psi} \cos 2 \alpha-A_{\phi}[F \cos 2 \alpha-J \sin 2 \alpha]+B_{\phi}\left(\frac{1}{2} F \sin 2 \alpha-J \sin ^{2} \alpha\right)-\frac{E B_{\psi}}{2} \sin 2 \alpha$

$w=\frac{1}{W}\left[\left(\frac{F}{W}\right)_{\phi}-\left(\frac{E}{W}\right)_{\psi}\right]$

$\frac{1}{J \mathrm{R}_{\mathrm{e}} \mathrm{P}_{\mathrm{r}}}\left[\left(\frac{G T_{\phi}-F T_{\psi}}{J}\right)_{\phi}+\left(\frac{E T_{\psi}-F T_{\phi}}{J}\right)_{\psi}\right]=-\frac{\mathrm{E}_{\mathrm{c}}}{4 \mu \mathrm{R}_{\mathrm{e}}}\left[B^{2}+4 A^{2}\right]+\frac{T_{\phi}}{J}$

$K=\frac{1}{W}\left[\left(\frac{W \Gamma_{11}^{2}}{E}\right)_{\psi}-\left(\frac{W \Gamma_{12}^{2}}{E}\right)_{\phi}\right]$

wherein $\phi$ and $\psi$ are considered as independent variables. This is a system of six equations in seven unknowns $E, F, G$ $, W, L, T$ and $q$. In equations $(26-29)$ the functions $A(\phi, \psi)$ and $B(\phi, \psi)$ are given by

$$
\begin{aligned}
A(\phi, \psi)=\mu\left[-\frac{(F \cos \alpha-J \sin \alpha)}{4 E^{2} J^{5}}\left\{E_{\phi}\left(2 E J^{3} \cos \alpha+F \sqrt{E} \sin \alpha\right)-4 E^{2} J^{2} J_{\phi} \cos \alpha-2 E \sqrt{E} F_{\phi} \sin \alpha+E \sqrt{E} E_{\psi} \sin \alpha\right\}\right. \\
\quad+\frac{\cos \alpha}{2 J^{3}}\left\{E_{\psi}(F \sin \alpha+J \cos \alpha)-2 E J_{\psi} \cos \alpha-E G_{\phi} \sin \alpha\right\}+\frac{(F \sin \alpha+J \cos \alpha)}{2 E J^{3}}\left\{\left(J E_{\phi}-2 E J_{\phi}\right) \sin \alpha\right. \\
\left.+\cos \alpha\left[-F E_{\phi}+2 E F_{\phi}-E E_{\psi}\right]\right\}-\frac{\sin \alpha}{2 J^{3}}\left\{\left(E_{\psi}(J \sin \alpha-F \cos \alpha)-2 E J_{\psi} \sin \alpha+E G_{\phi} \cos \alpha\right\}\right]
\end{aligned}
$$

$B(\phi, \psi)=\frac{4 \mu}{E J^{3}}\left[E_{\phi}(F \sin \alpha+J \cos \alpha)^{2}-2 E(F \sin \alpha+J \cos \alpha)\left(F_{\phi} \sin \alpha+J_{\phi} \cos \alpha\right)+E^{2}\left(J_{\psi} \sin 2 \alpha+G_{\phi} \sin ^{2} \alpha\right)\right]$

wherein

$\operatorname{Cos} \alpha=\frac{1}{\sqrt{E}}, \quad \operatorname{Sin} \alpha=\frac{\sqrt{E-1}}{\sqrt{E}} \quad \operatorname{Cos} 2 \alpha=\frac{2-E}{E}, \quad \operatorname{Sin} 2 \alpha=\frac{2 \sqrt{E-1}}{E}$

\section{Exact solutions}

Since our objective is to determine a class of exact solutions to flow equations for which the streamlines are characterized by equation (1) and to achieve it we set

$\phi=r(x, y)$

where

$x=r \operatorname{Cos} \theta, \quad y=r \operatorname{Sin} \theta$

Utilizing equations (34) and (35) in equations (25-33), we get

$$
\begin{aligned}
& q=\frac{\sqrt{E}}{W} \\
& -R_{e} w=R_{e} L_{\psi}-J A_{r}+\sqrt{E-1} A_{\psi}+B_{\psi} \\
& 0=-R_{e} L_{r}+\frac{A_{\psi}(2-E)}{J}+A_{r} \sqrt{E-1}-\frac{\sqrt{E-1} B_{\psi}}{J}
\end{aligned}
$$




$$
\begin{gathered}
J T_{r r}-2 \sqrt{E-1} T_{v r} v^{\prime}+\frac{E}{J} T_{v v}\left(v^{\prime}\right)^{2}+\left(J_{r}-\frac{E_{\psi}}{2 \sqrt{E-1}}-R_{e} P_{r}\right) T_{r}+\left(\frac{E_{\psi}}{J}-\frac{E_{r}}{2 \sqrt{E-1}}-\frac{E J_{\psi}}{J^{2}}+\frac{E}{J}\left(\frac{v^{\prime \prime}}{v^{\prime}}\right)\right) T_{v} v^{\prime} \\
=-\frac{J E_{c} P_{r}}{4 \mu}\left(B^{2}+4 A^{2}\right) \\
w=\left[\frac{f^{\prime}(r)}{r g(r)}+\frac{f^{\prime \prime}(r)}{g(r)}-\frac{2 f^{\prime}(r) g^{\prime}(r)}{g^{2}(r)}\right]\left(\frac{1}{v^{\prime}(\psi)}\right)+\left[\frac{g^{\prime}(r)}{r g(r)}+\frac{g^{\prime \prime}(r)}{g(r)}-\frac{2\left\{g^{\prime}(r)\right\}^{2}}{g^{2}(r)}\right]\left(\frac{v(\psi)}{v^{\prime}(\psi)}\right) \\
+\left[\frac{1}{r^{2} g^{2}(r)}+\frac{\left\{f^{\prime}(r)\right\}^{2}}{g^{2}(r)}\right]\left(\frac{v^{\prime \prime}(\psi)}{\left\{v^{\prime}(\psi)\right\}^{3}}\right)+\frac{2 f^{\prime}(r) g^{\prime}(r) v(\psi) v^{\prime \prime}(\psi)}{g^{2}(r)\left\{v^{\prime}(\psi)\right\}^{3}}+\frac{\left\{g^{\prime}(r)\right\}^{2} v^{2}(\psi) v^{\prime \prime}(\psi)}{g^{2}(r)\left\{v^{\prime}(\psi)\right\}^{3}}
\end{gathered}
$$

where

$$
\begin{aligned}
& A(r, \psi)=\frac{\mu}{J}\left[\frac{-2 J_{r} \sqrt{E-1}}{J}+\frac{E_{r}}{2 \sqrt{E-1}}+\frac{-(2-E) J_{\psi}}{J^{2}}\right] \\
& B(r, \psi)=4 \mu \frac{1}{J^{3}}\left[-J J_{r}+\sqrt{E-1} J_{\psi}\right] \\
& E=1+r^{2}\left[f^{\prime}(r)+g^{\prime}(r) v(\psi)\right]^{2} \\
& F=J \sqrt{E-1} \\
& G=r^{2} g^{2}(r) v^{\prime}(\psi)^{2} \\
& W=J=r g(r) v^{\prime}(\psi) \\
& \sqrt{E-1}=r f^{\prime}(r)+r g^{\prime}(r) v(\psi)
\end{aligned}
$$

In order to determine the solution of the flow equations we require an equation which the functions $f, g, v$ and the viscosity $\mu$ must satisfy for the class of flows under consideration and this is obtained by using the integrability condition $L_{r \psi}=L_{\psi r}$. The integrability condition, utilizing equations (43) and (46) yields

$$
r g v^{\prime} A_{r r}-2 r\left(f^{\prime}+g^{\prime} v\right) A_{r \psi}-\frac{\left[1-r^{2}\left(f^{\prime}+g^{\prime} v\right)^{2}\right]}{r g v^{\prime}} A_{\psi \psi}+g v^{\prime} A_{r}-A_{\psi}\left(\left(f^{\prime}+g^{\prime} v\right)+r\left(f^{\prime \prime}+g^{\prime \prime} v\right)\right)-\left\{B_{r}-\frac{\left(f^{\prime}+g^{\prime} v\right) B_{\psi}}{g v^{\prime}}\right\}_{\psi}=R_{e} w_{r}
$$

Once a solution of this equation is determined, the function $L$ and temperature distribution $T$ are determined from equations (37-38) and (39), respectively.

To determine the exact solutions of equation (48), the presence of term $\left(f^{\prime}+g^{\prime} v\right)$ in equation (48) and equation (2) suggests to consider the following two cases.

Case I $g=$ Constant

Case II $g \neq$ Constant

Case I: For the sake of simplicity we take $g=1$. On substituting $g=1$ in equations (36-42), in the absence of body force become

$$
\begin{aligned}
& q=\frac{\sqrt{1+\left(r f^{\prime}\right)^{2}}}{r v^{\prime}(\psi)}, \\
& w=\left[\frac{f^{\prime}(r)}{r}+f^{\prime \prime}(r)\right]\left(\frac{1}{v^{\prime}(\psi)}\right)+\left[\frac{1}{r^{2}}+\left\{f^{\prime}(r)\right\}^{2}\right]\left(\frac{v^{\prime \prime}(\psi)}{\left\{v^{\prime}(\psi)\right\}^{3}}\right), \\
& -\mathrm{R}_{\mathrm{e}} w=\mathrm{R}_{\mathrm{e}} v^{\prime} L_{v}-r v^{\prime}(\psi) A_{r}+r f^{\prime} v^{\prime} A_{v}+v^{\prime} B_{v},
\end{aligned}
$$


$0=-\mathrm{R}_{\mathrm{e}} L_{r}+r f^{\prime} A_{r}+\frac{\left\{1-\left(r f^{\prime}\right)^{2}\right\} A_{v}}{r}-f^{\prime} B_{v}$

$\left(r v^{\prime}(\psi)\right)^{3} T_{r r}-2\left(r v^{\prime}(\psi)\right)^{3} f^{\prime} T_{r v}+r\left(v^{\prime}(\psi)\right)^{3}\left\{1+\left(r f^{\prime}\right)^{2}\right\} T_{v v}+\left(r v^{\prime}(\psi)\right)^{2}\left\{v^{\prime}(\psi)-R_{e} P_{r}\right\} T_{r}$

$$
-r^{2}\left(v^{\prime}(\psi)\right)^{3}\left(r f^{\prime}\right)^{\prime} T_{v}=-\frac{E_{c} P_{r} J^{3}}{4 \mu}\left[B^{2}+4 A^{2}\right]
$$

$-r v^{\prime}(\psi) A_{r r}+2 r f^{\prime} v^{\prime}(\psi) A_{v r}+v^{\prime}(\psi)\left\{\frac{\left\{1-\left(r f^{\prime}\right)^{2}\right\}}{r}\right\} A_{v v}-v^{\prime}(\psi) f^{\prime} B_{v v}$

$$
+v^{\prime}(\psi) B_{v r}-v^{\prime}(\psi) A_{r}+v^{\prime}(\psi)\left(r f^{\prime}\right)^{\prime} A_{v}=-w, \mathrm{R}_{\mathrm{e}}
$$

where

$$
\begin{aligned}
& A(r, \psi)=\frac{\mu}{r v^{\prime}(\psi)}\left[r f^{\prime \prime}-f^{\prime}-\frac{\left(1-\left(r f^{\prime}\right)^{2}\right) v^{\prime \prime}(\psi)}{r\left(v^{\prime}(\psi)\right)^{2}}\right], \\
& B(r, \psi)=\frac{4 r \mu}{\left(r v^{\prime}(\psi)\right)^{3}}\left[-v^{\prime}(\psi)^{2}+r f^{\prime} v^{\prime \prime}(\psi)\right],
\end{aligned}
$$

The above system of equations indicates that its solutions strongly depend on the function $v(\psi)$ and its derivatives. Since $v^{\prime}(\psi) \neq 0$, therefore we consider the following cases

Case I (a) $v^{\prime \prime}(\psi)=0$

Case I(b) $v^{\prime \prime}(\psi) \neq 0$

Case I(a): $\quad v^{\prime \prime}=0$ gives

$v(\psi)=a \psi+b$

where $a \neq 0$ and $b$ are real constants.

Inserting equation (59) in equations (51-58) we get

$q=\frac{\sqrt{1+\left(r f^{\prime}\right)^{2}}}{a r}$,

$\mathrm{R}_{\mathrm{e}} a L_{v}=a r A_{r}-r f^{\prime} a A_{v}-a B_{v}-\frac{\mathrm{R}_{\mathrm{e}}\left(r f^{\prime \prime}+f^{\prime}\right)}{a r}$

$\operatorname{Re} L_{r}=r f^{\prime} A_{r}+\frac{\left(1-r^{2} f^{\prime 2}\right)}{r} A_{v}-f^{\prime} B_{v}$

$a r T_{r r}-2 a r f^{\prime} T_{v r}+\frac{a\left(1+r^{2} f^{\prime 2}\right)}{r} T_{v v}-a\left(r f^{\prime \prime}+f^{\prime}\right) T_{v}+\left(a-R_{e} P_{r}\right) T_{r}=\frac{-a r \mathrm{E}_{\mathrm{c}} \mathrm{P}_{\mathrm{r}}\left(B^{2}+4 A^{2}\right)}{4 \mu}$

$-r a A_{r r}+2 a r f^{\prime} A_{v r}+-a A_{r}+a A_{v}\left(r f^{\prime}\right)^{\prime}-a f^{\prime} B_{v v}+a B_{v r}=-w_{r} \mathrm{R}_{\mathrm{e}}$

$w=\frac{1}{a r}\left(r f^{\prime \prime}+f^{\prime}\right)$

where

$$
\begin{aligned}
& A=\frac{\mu}{a r}\left(r f^{\prime \prime}-f^{\prime}\right) \\
& B=\frac{-4 \mu}{a r^{2}}
\end{aligned}
$$


It is obvious from equation (64) that it is difficult to obtain exact solutions. However, we see that on eliminating $\mu$ from equations (66) and (67) the function $A$ can be eliminated from equation (64). On eliminating $\mu$, we get

$A=X(r) B$

where

$X(r)=\left(\frac{-1}{4}\right)\left(r^{2} f^{\prime \prime}-r f^{\prime}\right)$

provided $\left(r^{2} f^{\prime \prime}-r f^{\prime}\right) \neq 0$.

Inserting equation (68) in equation (64) we get

$$
\begin{gathered}
a r X B_{r r}-a(1+2 M X) B_{v r}+\frac{a\left\{M-X\left(1-M^{2}\right)\right\}}{r} B_{v v}+a B_{r}\left\{2 r X^{\prime}+X\right\} \\
-a\left(2 M X^{\prime}+M^{\prime} X\right) B_{v}+a B\left(r X^{\prime}\right)^{\prime}={ }_{R_{e}}\left(\frac{M^{\prime}}{a r}\right)^{\prime}
\end{gathered}
$$

where

$M(r)=r f^{\prime}(r)$

The form of equation (70) suggests to seek a solution of the form

$B=S(r)+K(r) v(\psi)$

where $S(r)$ and $K(r)$ are to be determined.

Substituting equation (72) in equation (70) we get

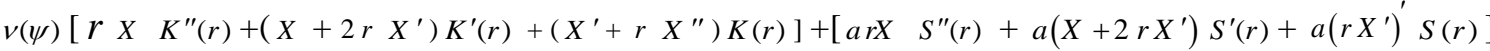

$$
\begin{aligned}
& =R_{e}\left(\frac{M^{\prime}}{a r}\right)^{\prime}+a(1+2 M X) K^{\prime}(r)+a\left(2 M X^{\prime}+M^{\prime} X\right) K(r)
\end{aligned}
$$

As $r$ and $\psi$ are independent variables therefore equation (73) provides

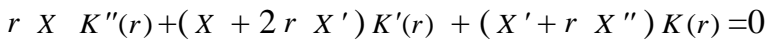

and

$a r X \quad S^{\prime \prime}(r)+a\left(X+2 r X^{\prime}\right) S^{\prime}(r)+a\left(r X^{\prime}\right)^{\prime} S(r)=Z_{1}(r)$

where

$Z_{1}(r)=R_{e}\left(\frac{M^{\prime}}{a r}\right)^{\prime}+a(1+2 M X) K^{\prime}(r)+a\left(2 M X^{\prime}+M^{\prime} X\right) K(r)$

Equation (74) can be rewritten as

$\left[\begin{array}{ll}r\left(\begin{array}{ll}X & K\end{array}\right)^{\prime} & ]^{\prime}=0\end{array}\right.$

Equation (77) yields 
$K(r)=\frac{k_{1} \ln r}{X(r)}+\frac{k_{2}}{X(r)}$

where $k_{1}$ and $k_{2}$ are constants.

Also equation (75) can be rewritten as

$\left[r(X S)^{\prime}\right]^{\prime}=Z_{1}(r)$

Whose solution is

$S(r)=\frac{1}{X} \int\left[\frac{1}{r} \int Z_{1}(r) d r\right] d r+\frac{k_{3} \ln r+k_{4}}{X}$

where $k_{3}$ and $k_{4}$ are constants.

On substituting equation (78) and equation (80) in equation (72) we get

$B=\frac{1}{X} \int\left[\frac{1}{r} \int Z_{1}(r) d r\right] d r+\frac{k_{3} \ln r+k_{4}}{X}+\left(\frac{k_{1} \ln r+k_{2}}{X}\right) v(\psi)$

The expression for viscosity employing equation (66) or equation (67) is

$\mu(r, \psi)=\frac{-a r^{2}}{4}\left[\frac{1}{X} \int\left[\frac{1}{r} \int Z_{1}(r) d r\right] d r+\frac{k_{3} \ln r+k_{4}}{X}+\left(\frac{k_{1} \ln r+k_{2}}{X}\right) v(\psi)\right]$

The expression of function $L$ is determined by finding the solution of equation (61) and (62). The solution, employing equations (81) and (68), is

$$
\begin{aligned}
a R_{e} L=-R_{e}\left(\frac{M^{\prime}}{a r}\right) & v+a r(S X)^{\prime} v-a K(M X+1) v+a r(K X)^{\prime}\left(\frac{v^{2}}{2}\right) \\
& +a \int M(S X)^{\prime} d r+\int\left[\frac{K}{r}\left\{a X\left(1-M^{2}\right)-a M\right\}\right] d r+p_{1}
\end{aligned}
$$

The temperature distribution $T$ is determined from equation (64). Equation (64) utilizing equations (68), (81) and (82) becomes

$a r T_{r r}-2 a^{2} r f^{\prime} T_{v r}+\frac{a\left(1+r^{2} f^{\prime 2}\right)}{r} T_{v v}-a\left(r f^{\prime \prime}+f^{\prime}\right) T_{v}+\left(a-R_{e} P_{r}\right) T_{r}=\frac{\mathrm{E}_{\mathrm{c}} \mathrm{P}_{\mathrm{r}}\left(1+4 X^{2}\right)}{r}\{S(r)+K(r) v\}$

Right-hand side of equation (84) suggests to seek a solution of the form

$T=T_{1}(r)+T_{2}(r) v(\psi)$

On using equation (85) into equation (84), we get

$$
\begin{aligned}
& T_{1}^{\prime \prime}+\frac{\left(a-R_{e} P_{r}\right)}{a r} T_{1}^{\prime}=Z_{2}(r) \\
& T_{2}^{\prime \prime}+\frac{\left(a-R_{e} P_{r}\right)}{a r} T_{2}^{\prime}=Z_{3}(r)
\end{aligned}
$$

where

$$
\begin{aligned}
& Z_{2}(r)=\frac{\mathrm{E}_{\mathrm{c}} \mathrm{P}_{\mathrm{r}}\left(1+4 X^{2}\right)}{a r^{2}} S(r)+2 a^{2} r f^{\prime} T_{2}^{\prime}+a\left(r f^{\prime \prime}+f^{\prime}\right) T_{2} \\
& Z_{3}(r)=\frac{\mathrm{E}_{\mathrm{c}} \mathrm{P}_{\mathrm{r}}\left(1+4 X^{2}\right)}{a r^{2}} K(r)
\end{aligned}
$$


It is obvious from equation (86) and (87) that their solutions depend on the value of $\left(a-R_{e} P_{r}\right)$. The solution of equations (86) and (87) when $\left(a-R_{e} P_{r}\right) \neq 0$ is

$$
\begin{aligned}
& T_{1}(r)=\int r^{\frac{-\left(a-R_{e} P_{r}\right)}{a}}\left[\int\left\{r^{\frac{\left(a-R_{e} P_{r}\right)}{a}} Z_{2}(r)\right\} d r\right] d r+H_{3} \int r^{\frac{-\left(a-R_{e} P_{r}\right)}{a}} d r+H_{4} \\
& T_{2}(r)=\int r^{\frac{-\left(a-R_{e} P_{r}\right)}{a}}\left[\int\left\{r^{\frac{\left(a-R_{e} P_{r}\right)}{a}} Z_{3}(r)\right\} d r\right] d r+H_{1} \int r^{\frac{-\left(a-R_{e} P_{r}\right)}{a}} d r+H_{2}
\end{aligned}
$$

Now when $\left(a-R_{e} P_{r}\right)=0$ the solution of equations (86) and (89) is

$$
\begin{aligned}
& T_{1}(r)=\iint Z_{2}(r) d r d r+H_{5} r+H_{6} \\
& T_{2}(r)=\iint Z_{3}(r) d r d r+H_{7} r+H_{8}
\end{aligned}
$$

where $H_{i}, i=1,2, \ldots 8$ are constant.

On eliminating $\mu$ from (66) and (67), the equation (69) indicates that it is valid only for $\left(r f^{\prime \prime}-f^{\prime}\right) \neq 0$. When $\left(r f^{\prime \prime}-f^{\prime}\right)=0$, the function $A=0$ and we get

$f(r)=\frac{1}{2} c_{1} r^{2}+c_{2}$

Equation (64) on substituting $A=0$

$c_{1} r B_{v v}-B_{r v}=0$

The solution of equation (95) is

$B=\frac{1}{2} c_{1} b_{1} r^{2}+b_{1} v(\psi)+\int I(r) d r+c_{3}$

In above $c_{1} \neq 0, c_{2}, c_{3}$ and $b_{1}$ are constants. The function $I(r)$ is an unknown function. Utilizing equation (96) in equation (67) we get

$\mu=\frac{-a r^{2}}{4}\left[c_{3}+b_{1} v(\psi)+\frac{1}{2} c_{1} b_{1} r^{2}+\int I(r) d r\right]$

The solution of equations (61) and (62), for $f(r)=\frac{1}{2} c_{1} r^{2}+c_{2}$ is

$\operatorname{Re} L=\left(-b_{1}-\frac{2 c_{1} \operatorname{Re}}{a^{2}}\right) v-\frac{1}{2} c_{1} b_{1} r^{2}+M_{0}$

where $M_{0}$ is a real constant

The energy equation (63) employing equations (96) and (97), becomes

$a r^{2} T_{r r}-2 c_{1} a r^{3} T_{v r}+a r\left(1+c_{1}^{2} r^{4}\right) T_{v V}-2 c_{1} a r^{2} T_{v}+r\left(a-R_{e} P_{r}\right) T_{r}=\mathrm{E}_{\mathrm{c}} \mathrm{P}_{\mathrm{r}}\left[c_{3}+b_{1} v(\psi)+\frac{1}{2} c_{1} b_{1} r^{2}+\int I(r) d r\right]$

Equation (99) suggests to seek a solution of the form

$T=A_{1} v^{3}(\psi)+R_{2}(r) v^{2}(\psi)+R_{1}(r) v(\psi)+R_{0}(r)$

Equation (99), employing equation (100), provides

$a r^{2} R_{2}^{\prime \prime}+r\left(a-R_{e} P_{r}\right) R_{2}^{\prime}=6 a c_{1} A_{1} r^{2}$ 
$a r^{2} R_{1}^{\prime \prime}+r\left(a-R_{e} P_{r}\right) R_{1}^{\prime}=4 a c_{1} r^{3} R_{2}^{\prime}+4 a c_{1} r^{2} R_{2}-6 a A_{1} r\left(1+c_{1}^{2} r^{4}\right)+b_{1} E_{c} P_{r}$

$a r^{2} R_{0}^{\prime \prime}+r\left(a-R_{e} P_{r}\right) R_{0}^{\prime}=2 a c_{1} r^{3} R_{1}^{\prime}+2 a c_{1} r^{2} R_{1}-2 a r\left(1+c_{1}^{2} r^{4}\right) R_{2}+E_{c} P_{r}\left(c_{3}+\frac{1}{2} c_{1} b_{1} r^{2}+\int I(r) d r\right)$

When $\left(a-R_{e} P_{r}\right) \neq 0$ the equations $(101-103)$ give

$$
\begin{gathered}
R_{2}(r)=\frac{3 a A_{1} c_{1} r^{2}}{\left(2 a-R_{e} P_{r}\right)}+\frac{a n_{1}}{R_{e} P_{r}} r^{\frac{R_{e} P_{r}}{a}}+n_{2},\left(2 a-R_{e} P_{r}\right) \neq 0 \\
R_{1}(r)=-\frac{6 a A_{1} c_{1}^{2} r^{5}}{5\left(5 a-R_{e} P_{r}\right)}-\frac{6 a A_{1} r}{\left(a-R_{e} P_{r}\right)}+\frac{9 a^{2} A_{1} c_{1}^{2} r^{4}}{\left(4 a-R_{e} P_{r}\right)\left(2 a-R_{e} P_{r}\right)}+\frac{2 a c_{1} r^{2} n_{2}}{\left(2 a-R_{e} P_{r}\right)}+\frac{a n_{1}}{R_{e} P_{r}} r^{\frac{R_{e} P_{r}}{a}}+n_{4}-\frac{b_{1} E_{c} \ln r}{R_{e}} \\
+\frac{a n_{1} r^{\frac{R_{e} P_{r}}{a}}\left[2 a\left(c_{1} r^{2} n_{1}+n_{3}\right)+R_{e} P_{r}\left(2 c_{1} r^{2} n_{1}+n_{3}\right)\right]}{R_{e} P_{r}\left(2 a-R_{e} P_{r}\right)},
\end{gathered}
$$

where $\left(2 a-R_{e} P_{r}\right) \neq 0,\left(4 a-R_{e} P_{r}\right) \neq 0,\left(5 a-R_{e} P_{r}\right) \neq 0$

$$
R_{0}(r)=n_{6}+\int M_{1}(r) d r
$$

$$
\begin{aligned}
& M_{1}(r)=n_{5} r^{\left\{\frac{\left(a-R_{e} P_{r}\right)}{a}\right\}}+r^{-\left\{\frac{\left(a-R_{e} P_{r}\right)}{a}\right\}} \int\left\{E_{c} P_{r}\left(c_{3}+\frac{1}{2} c_{1} b_{1} r^{2}+\int I(r) d r\right)\right\} r^{\left\{\frac{\left(a-R_{e} P_{r}\right)}{a}\right\}} d r
\end{aligned}
$$

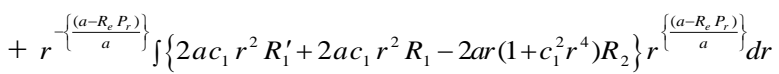

and $n_{1}, n_{2}, n_{3}, n_{4}, n_{5}, n_{6}$ are non-zero constants.

When $\left(a-R_{e} P_{r}\right)=0$, equations $(101-103)$ yield

$$
\begin{aligned}
& R_{2}(r)=3 c_{1} A_{1} r^{2}+n_{7} r+n_{8} \\
& R_{1}(r)=\frac{-6 A_{1} c_{1}^{2}}{20} r^{5}+3 c_{1}^{2} A_{1} r^{4}+\frac{4}{3} c_{1} n_{7} r^{3}+2 c_{1} n_{8} r^{2}-6 A_{1} r \ln r+\left(6 A_{1}+n_{9}\right) r-\frac{b_{1} E_{c} P_{r}}{a} \ln r+n_{10} \\
& R_{0}(r)=\iint M_{2}(r) d r d r+n_{11} r+n_{12}
\end{aligned}
$$

where

$M_{2}(r)=2 c_{1} r R_{1}^{\prime}(r)+2 c_{1} R_{1}(r)-\frac{2}{r}\left(1+c_{1}^{2} r^{4}\right) R_{2}(r)+\frac{E_{c} P_{r}}{a r^{2}}\left(c_{3}+\frac{c_{1} b_{1}}{2} r^{2}+\int I(r) d r\right)$

and $n_{7}, n_{8} n_{9}, n_{10} n_{11}, n_{12}$ are non-zero constants.

Case I(b):

In this case $v^{\prime \prime} \neq 0$, the equations (57) and (58) can be rewritten as

$$
\begin{aligned}
& A=\frac{\mu}{r^{2} v^{\prime}}\left[r M^{\prime}-2 M-\left(1-M^{2}\right)\left(\frac{v^{\prime \prime}}{v^{\prime 2}}\right)\right] \\
& B=\frac{4 \mu}{r^{2} v^{\prime}}\left[-1+M\left(\frac{v^{\prime \prime}}{v^{\prime 2}}\right)\right]
\end{aligned}
$$

where

$$
M=r f^{\prime}
$$

Equations (112) and (113) indicate that we can eliminate $\mu$ from these equations if we set 
$\frac{v^{\prime \prime}}{v^{\prime 2}}=1$

Equation (115) gives

$v=\ln \left[\frac{-1}{\left(c_{4} \psi+c_{5}\right)}\right]$

where $c_{4} \neq 0$ and $c_{5}$ are constants.

On eliminating $\mu$ from equations (112) and (113), employing (115), we get

$B=Y(r) A$

where

$Y(r)=\frac{4(-1+M)}{r M^{\prime}-2 M-\left(1-M^{2}\right)}, \quad M \neq 1$

Equation (56) employing equations (115) and (117), become

$r A_{r r}-(2 M+Y) A_{v r}+\left(\frac{M Y-\left(1-M^{2}\right)}{r}\right) A_{\nu v}+A_{r}-\left(M^{\prime}+Y^{\prime}\right) A_{v}=R_{e}\left(\frac{e^{-2 v}}{c_{4}^{2}}\right)\left[\frac{M^{\prime}}{r}+\frac{\left(1+M^{2}\right)}{r^{2}}\right]^{\prime}$

Equation (119) suggests to seek solution of the form

$A=C(r, v)+e^{-2 v} D(r)$

where the functions $C(r, v)$ and $D(r)$ are to be determined. Substituting equation (120) in equation (119) we get

$$
\begin{gathered}
r C_{r r}-(2 M+Y) C_{r v}+\left(\frac{M Y-\left(1-M^{2}\right)}{r}\right) C_{v v}+C_{r}-\left(M^{\prime}+Y^{\prime}\right) C_{v} \\
+e^{-2 v}\left[r D^{\prime \prime}+2(2 M+Y) D^{\prime}+4\left(\frac{M Y-\left(1-M^{2}\right)}{r}\right) D+D^{\prime}+2\left(M^{\prime}+Y^{\prime}\right) D\right] \\
=R_{e}\left(\frac{e^{-2 v}}{c_{4}^{2}}\right)\left[\frac{M^{\prime}}{r}+\frac{\left(1+M^{2}\right)}{r^{2}}\right]^{\prime}
\end{gathered}
$$

Which on equating the coefficients of $e^{-2 v}$ gives

$$
\begin{aligned}
& r C_{r r}-(2 M+Y) C_{r v}+\left(\frac{M Y-\left(1-M^{2}\right)}{r}\right) C_{v v}+C_{r}-\left(M^{\prime}+Y^{\prime}\right) C_{v}=0 \\
& r^{2} D^{\prime \prime}+r(4 M+2 Y+1) D^{\prime}+\left[4 M Y-4\left(1-M^{2}\right)+2 r\left(M^{\prime}+Y^{\prime}\right)\right] D=\left(\frac{R_{e}}{c_{4}^{2}}\right) r\left[\frac{M^{\prime}}{r}+\frac{\left(1+M^{2}\right)}{r^{2}}\right]^{\prime}
\end{aligned}
$$

The equation (123) can be reduced to Cauchy equation if we set

$$
(4 M+2 Y+1)=m_{1}
$$

and

$4 M Y-4\left(1-M^{2}\right)+2 r\left(M^{\prime}+Y^{\prime}\right)=m_{2}$

The solution of the system of equations (119), (124) and (125) is

$M=-1, m_{1}=-11, m_{2}=16$ 
Equation (123), utilizing equation (126), becomes

$r^{2} D^{\prime \prime}-11 r D^{\prime}+16 D=\left(\frac{R_{e}}{c_{4}^{2}}\right)\left[\frac{-4}{r^{2}}\right]$

The solution of equation (127) is

$D(r)=D_{1} r^{(6+2 \sqrt{5})}+D_{2} r^{(6-2 \sqrt{5})}+\left(\frac{-R_{e}}{11 c_{4}^{2}}\right)\left(\frac{1}{r^{2}}\right)$

where $D_{1}$ and $D_{2}$ are constants.

Equation (122), utilizing equation (126) becomes

$r^{2} C_{r r}+6 r C_{r v}+4 C_{v V}+r C_{r}=0$

Equation (129) indicates to seek a solution of the form

$C=C_{1}(r)+S_{1}(v)+C_{2}(r) S_{2}(v)$

Substituting equation (130) in equation (129)

$\left\{r\left(r C_{1}^{\prime}\right)^{\prime}\right\}+4 S_{1}^{\prime \prime}+\left\{4 C_{2} S_{2}^{\prime \prime}+6 r C_{2}^{\prime} S_{2}^{\prime}+r\left(r C_{2}^{\prime}\right)^{\prime} S_{2}\right\}=0$

Differentiating equation (131) with respect to " $r$ " we get

$\left[r\left(r C_{1}^{\prime}\right)^{\prime}\right]^{\prime}+4 C_{2}^{\prime} S_{2}^{\prime \prime}+6\left(r C_{2}^{\prime}\right)^{\prime} S_{2}^{\prime}+\left[r\left(r C_{2}^{\prime}\right)^{\prime}\right]^{\prime} S_{2}=0$

Differentiating equation (132) with respect to " $v$ " we obtain

$4 C_{2}^{\prime} S_{2}^{\prime \prime \prime}+6 J S_{2}^{\prime \prime}+[r J]^{\prime} S_{2}^{\prime}=0$

where

$J(r)=\left(r C_{2}^{\prime}(r)\right)^{\prime}$

Equation (133) can be written as

$4 C_{2}^{\prime}\left(\frac{Z^{\prime \prime}}{Z}\right)+6 J\left(\frac{Z^{\prime}}{Z}\right)+[r J]^{\prime}=0$

where

$Z(v)=S_{2}^{\prime}(v)$

Differentiating equation (134) with respect to " $v$ " and arranging the terms we obtain

$\frac{\left(\frac{Z^{\prime \prime}}{Z}\right)^{\prime}}{\left(\frac{Z^{\prime}}{Z}\right)^{\prime}}=-\frac{3}{2} \frac{J(r)}{C_{2}^{\prime}(r)}=d_{1}$

where $d_{1}$ is a non-zero arbitrary constant. Equation (138) provides

$J(r)=-\frac{2}{3} d_{1} C_{2}^{\prime}(r)$ 
$Z^{\prime \prime}=d_{1} Z^{\prime}+d_{2} Z$

where $d_{2}$ is a constant

Equation (138) on utilizing equation (134) and integrating once gives

$r C_{2}^{\prime}(r)+\frac{2}{3} d_{1} C_{2}(r)=d_{3}$

where $d_{3}$ is a constant. The solution of equation (140) is

$C_{2}(r)=\frac{3 d_{3}}{2 d_{1}}+d_{4} r^{-2 d_{1} / 3}$

where $d_{4}$ is constant.

On substituting equation (136) in equation (139) and integrating once, we obtain

$S_{2}^{\prime \prime}(v)-d_{1} S_{2}^{\prime}(v)-d_{2} S_{2}(v)=d_{5}$

where $d_{5}$ is a constant. Now inserting equation (141) and equation (142) in equation (134), we obtain

$C_{2}^{\prime}\left\{S_{2}^{\prime \prime \prime}-d_{1} S_{2}^{\prime \prime}+\frac{d_{1}^{2}}{9} S_{2}^{\prime}\right\}=0$

As $C_{2}^{\prime}(r) \neq 0$, equation (143) gives

$S_{2}^{\prime \prime \prime}-d_{1} S_{2}^{\prime \prime}+\frac{d_{1}^{2}}{9} S_{2}^{\prime}=0$

which can be rewritten as

$\left(S_{2}^{\prime \prime}-d_{1} S_{2}^{\prime}\right)^{\prime}+\frac{d_{1}^{2}}{9} S_{2}^{\prime}=0$

Employing equation (141) in equation (145), we find

$d_{2}=-\frac{d_{1}^{2}}{9}$

Inserting equations (145) and (146) in equation (132), we get

$r\left(r C_{1}^{\prime}\right)^{\prime}=-4 C_{2} d_{5}+d_{6}$

whose solution is

$C_{1}(r)=-4 d_{5} \int\left[\frac{1}{r} \int \frac{C_{2}(r)}{r} d r\right] d r+d_{6} \int \frac{\ln r}{r} d r+d_{7} \ln r+d_{8}$

where $d_{5}, d_{6}, d_{7}$, and $d_{8}$ are constants.

Now the equations (142), (143) and (146) must satisfy equation (131), and therefore we get

$S_{1}=-\frac{d_{3}}{6} \int\left\{\int\left[9 S_{2}^{\prime}(v)-d_{1} S_{2}(v)\right] d v\right\} d v-\frac{d_{6}}{8} v^{2}+d_{9} v+d_{11}$

The solution of equation (145) is 
$S_{2}(v)=d_{12} \operatorname{Exp}\left[\frac{(3+\sqrt{5})}{6} d_{1} v\right]+d_{13} \operatorname{Exp}\left[\frac{(3-\sqrt{5})}{6} d_{1} v\right]+\frac{9 d_{11}}{d_{1}^{2}}$

which is the solution of equation (143). In above equations $d_{9}, . ., d_{11}, d_{12}$ and $d_{13}$ are constants of integration.

On substituting the values $C_{1}(r), C_{2}(r), S_{1}(v), S_{2}(v)$ and $D(r)$ in equation (120) we get

$$
\begin{gathered}
A=-\frac{3 d_{3} d_{5}}{d_{1}}(\ln r)^{2}-\frac{9 d_{4} d_{5}}{d_{1}^{2}} r^{-2 d_{1} / 3}+d_{6} \frac{(\ln r)^{2}}{2}+d_{7} \ln r+d_{8}-\frac{d_{3}}{6} \int\left\{\int\left[9 S_{2}^{\prime}(v)-d_{1} S_{2}(v)\right] d v\right\} d v-\frac{d_{6}}{8} v^{2}+d_{9} v+d_{10} \\
+\left\{\frac{3 d_{3}}{2 d_{1}}+d_{4} r^{-2 d_{1} / 3}\right\}\left\{d_{12} \operatorname{Exp}\left[\frac{(3+\sqrt{5})}{6} d_{1} v\right]+d_{13} \operatorname{Exp}\left[\frac{(3-\sqrt{5})}{6} d_{1} v\right]+\frac{9 d_{11}}{d_{1}^{2}}\right\} \\
+e^{-2 v}\left\{D_{1} r^{(6+2 \sqrt{5})}+D_{2} r^{(6-2 \sqrt{5})}+\left(\frac{-R_{e}}{11 c_{4}^{2}}\right)\left(\frac{1}{r^{2}}\right)\right\}
\end{gathered}
$$

The viscosity distribution from equation (57) or (58) is

$$
\begin{gathered}
\mu=\left(\frac{-r^{2} c_{4} e^{v}}{2}\right)\left[-\frac{3 d_{3} d_{5}}{d_{1}}(\ln r)^{2}-\frac{9 d_{4} d_{5}}{d_{1}^{2}} r^{-2 d_{1} / 3}+d_{6} \frac{(\ln r)^{2}}{2}+d_{7} \ln r+d_{8}-\frac{d_{3}}{6} \int\left\{\int\left[9 S_{2}^{\prime}(v)-d_{1} S_{2}(v)\right] d v\right\} d v-\frac{d_{6}}{8} v^{2}+d_{9} v+d_{10}\right. \\
+\left\{\frac{3 d_{3}}{2 d_{1}}+d_{4} r^{-2 d_{1} / 3}\right\}\left\{d_{12} \operatorname{Exp}\left[\frac{(3+\sqrt{5})}{6} d_{1} v\right]+d_{13} \operatorname{Exp}\left[\frac{(3-\sqrt{5})}{6} d_{1} v\right]+\frac{9 d_{11}}{d_{1}^{2}}\right\} \\
\left.+e^{-2 v}\left\{D_{1} r^{(6+2 \sqrt{5})}+D_{2} r^{(6-2 \sqrt{5})}+\left(\frac{-R_{e}}{11 c_{4}^{2}}\right)\left(\frac{1}{r^{2}}\right)\right\}\right]
\end{gathered}
$$

The solution of equations (53-54), utilizing equations (112-113), (116),(126) and (152) is

$R_{e} L=-\left[C_{1}(r)+S_{1}(v)+C_{2}(r) S_{2}(v)+e^{-2 v} D(r)\right]-4 S_{1}^{\prime} \ln r-4 S_{2}^{\prime} \int \frac{C_{2}}{r} d r+8 e^{-2 v} \int \frac{D}{r} d r+\left(d_{7}+6 d_{9}\right) v-\frac{3 d_{6}}{4} v^{2}+p_{4}$

provided

$d_{3}=0, \quad d_{5}=d_{11}$

Now for this case the equation for $T$ is

$$
\begin{aligned}
& r^{2} T_{r r}+2 r T_{v r}+\frac{2}{r} T_{v v}\left(v^{\prime}\right)+r\left(1-\frac{R_{e} P_{r}}{c_{4}} e^{-v}\right) T_{r} \\
&=\frac{10 E_{c} P_{r}}{c_{4}}\left[e^{-v}\left\{d_{6} \frac{(\ln r)^{2}}{2}+d_{7} \ln r+d_{8}\right\}+e^{-v}\left\{-\frac{d_{6}}{8} v^{2}+d_{9} v+d_{10}\right\}\right. \\
&+e^{-v}\left\{d_{4} r^{-2 d_{1} / 3}\right\}\left\{d_{12} \operatorname{Exp}\left[\frac{(3+\sqrt{5})}{6} d_{1} v\right]+d_{13} \operatorname{Exp}\left[\frac{(3-\sqrt{5})}{6} d_{1} v\right]\right\} \\
&+e^{-3 v}\left(\frac{-R_{e}}{11 c_{4}^{2}}\right)\left(\frac{1}{r^{2}}\right)+e^{-3 v}\left\{D_{1} r^{(6+2 \sqrt{5})}+D_{2} r^{(6-2 \sqrt{5})}\right\}
\end{aligned}
$$

It is obvious from the equation (155) that it is extremely difficult to determine the exact solution of equation (155). However, we obtained by setting

$$
T=e^{-v} T_{1}(r)+S_{3}(v)+r^{b} S_{4}(v)
$$

in the temperature equation (155). Inserting equation (156) in equation (155) and arranging the terms we get

$$
\begin{gathered}
e^{-v}\left[r^{2} T_{1}^{\prime \prime}(r)-r T_{1}^{\prime}(r)+2 T_{1}(r)\right]+r^{b}\left[b(b-1) S_{4}(v)+2 b S_{4}^{\prime}(v)+2 S_{4}^{\prime \prime}(v)+b r^{b}\left(1-\frac{R_{e} P_{r}}{c_{4}} e^{-v}\right) S_{4}(v)\right]+2 S_{3}^{\prime \prime}(v)-\frac{R_{e} P_{r}}{c_{4}} e^{-2 v} r T_{1}^{\prime}(r) \\
=\frac{10 E_{c} P_{r}}{c_{4}}\left[e^{-v}\left\{d_{6} \frac{(\ln r)^{2}}{2}+d_{7} \ln r+d_{8}\right\}+e^{-v}\left\{-\frac{d_{6}}{8} v^{2}+d_{9} v+d_{10}\right\}\right. \\
+e^{-v}\left\{d_{4} r^{-2 d_{1} / 3}\right\}\left\{d_{12} \operatorname{Exp}\left[\frac{(3+\sqrt{5})}{6} d_{1} v\right]+d_{13} \operatorname{Exp}\left[\frac{(3-\sqrt{5})}{6} d_{1} v\right]\right\} \\
+e^{-3 v}\left(\frac{-R_{e}}{11 c_{4}^{2}}\right)\left(\frac{1}{r^{2}}\right)+e^{-3 v}\left\{D_{1} r^{(6+2 \sqrt{5})}+D_{2} r^{(6-2 \sqrt{5})}\right\}
\end{gathered}
$$


Comparing terms of right-hand side and left-hand side of equation (157) we get

$$
\begin{aligned}
& T_{1}(r)=\frac{5 d_{8} E_{c} P_{r}}{c_{4}} \\
& S_{3}^{\prime \prime}(v)=\frac{5 E_{c} P_{r}}{c_{4}}\left(d_{9} v+d_{10}\right) e^{-v}
\end{aligned}
$$

and

$$
S_{4}^{\prime \prime}(v)-2 S_{4}^{\prime}(v)+\left(2+\frac{R_{e} P_{r}}{c_{4}} e^{-v}\right) S_{4}(v)=\frac{5 E_{c} P_{r}}{c_{4}} d_{4}\left\{d_{12} e^{\frac{(1+\sqrt{5})}{2} v}+d_{13} e^{\frac{(1-\sqrt{5})}{2} v}\right\}+e^{-3 v}\left(\frac{-R_{e}}{11 c_{4}^{2}}\right)
$$

provided

$D_{1}=D_{2}=d_{6}=d_{7}=0, d_{1}=3, b=-2$,

Solution of equation (159) and (160) is

$$
\begin{aligned}
& S_{3}(v)=\frac{5 E_{c} P_{r}}{c_{4}} \iint\left(d_{9} v+d_{10}\right) e^{-v} d v+s_{1} v+s_{2} \\
& \left.\left.S_{4}(v)=\frac{C_{1}}{A 1} e^{v} \text { Gamma[1-2i }\right] \text { BesselJ }\left[-2 i, 2 \sqrt{A 1 e^{-v}}\right]+\frac{C_{2}}{A 1} e^{v} \text { Gamma[1+2i }\right] \operatorname{BesselJ}\left[2 i, 2 \sqrt{A 1 e^{-v}}\right] \\
& +\frac{i}{2} e^{v} \text { Gamma[1-2i]Gamma[1+2i] } \\
& \left\{\operatorname{BesselJ}\left[2 i, 2 \sqrt{A 1 e^{-v}}\right] \int e^{-4 v}\left\{A 4+A 3 e^{(7-\sqrt{5}) v / 2}+A 2 e^{(7+\sqrt{5}) v / 2}\right\} \operatorname{BesselJ}\left[-2 i, 2 \sqrt{A 1 e^{-v}}\right] d v\right. \\
& \left.-\operatorname{BesselJ}\left[-2 i, 2 \sqrt{A 1 e^{-v}}\right] \int e^{-4 v}\left\{A 4+A 3 e^{(7-\sqrt{5}) / 2}+A 2 e^{(7+\sqrt{5}) v / 2}\right\} \operatorname{BesselJ}\left[2 i, 2 \sqrt{A 1 e^{-v}}\right] d v\right\}
\end{aligned}
$$

where

$$
A 1=\frac{R_{e} P_{r}}{c_{4}}, \quad A 2=\frac{5 E_{c} P_{r} d_{4} d_{12}}{c_{4}}, \quad A 3=\frac{5 E_{c} P_{r} d_{4} d_{13}}{c_{4}}, \quad A 4=-\frac{R_{e}}{11 c_{4}^{2}}
$$

The solution (163) of equation (160) is obtained using Mathematica.

We know that equation (118) does not hold for $M=1$. To determine the solution we have to use equation (112) and (113) which on substituting $M=1$, give

$$
B=0
$$

$A=\frac{-2 \mu}{r^{2} v^{\prime}}$

Equation (56), utilizing equation (165) and $M=1$ becomes

$$
r^{2} A_{r r}-2 r A_{v r}+r A_{r}=R_{e}\left(\frac{e^{-2 v}}{c_{4}^{2}}\right)\left(\frac{-4}{r^{2}}\right)
$$

Following the previous case $M \neq 1$, we seek a solution of the form

$$
A=N(r, v)+e^{-2 v} D_{1}(r)
$$

Inserting Equation (168) in equation (167) gives

$$
\left\{r^{2} N_{r r}-2 r N_{v r}+r N_{r}\right\}+e^{-2 v}\left\{r^{2} D_{1}^{\prime \prime}+5 r D_{1}^{\prime}\right\}=R_{e}\left(\frac{e^{-2 v}}{c_{4}^{2}}\right)\left(\frac{-4}{r^{2}}\right)
$$


Which on comparing left hand side and right hand side give

$$
\begin{aligned}
& r^{2} D_{1}^{\prime \prime}+5 r D_{1}^{\prime}=\left(\frac{R_{e}}{c_{4}^{2}}\right)\left(\frac{-4}{r^{2}}\right) \\
& r^{2} N_{r r}-2 r N_{v r}+r N_{r}=0
\end{aligned}
$$

The solution of equation of (171) is

$$
D_{1}(r)=\frac{R_{e}}{c_{4}^{2}}\left(\frac{1}{r^{2}}\right)-\frac{4 d_{14}}{r^{4}}+d_{15}
$$

Left- hand side of equation (172) suggest to seek a solution of the form

$$
N=C_{3}(r)+S_{5}(v)+C_{4}(r) S_{6}(v)
$$

which on substituting in equation (171) gives

$\left\{r^{2} C_{3}^{\prime \prime}+r C_{3}^{\prime}\right\}+S_{6}\left\{r^{2} C_{4}^{\prime \prime}+r C_{4}^{\prime}\right\}-2 r C_{4}^{\prime} S_{6}^{\prime}=0$

Differentiating equation (175) with respect to $v$ and rearranging the terms of $r$ and $v$ we get

$$
r C_{4}^{\prime \prime}(r)+\left(1-d_{16}\right) C_{4}^{\prime}(r)=0
$$

$S_{6}^{\prime \prime}(v)-\frac{d_{16}}{2} S_{6}^{\prime}(v)=0$

where $d_{14}$ is a separation constant. Solution of equations (175) and (176) are

$$
\begin{aligned}
& C_{4}(r)=\frac{d_{17}}{d_{16}} r^{d_{16}}+d_{18} \\
& S_{6}(v)=\frac{-2 d_{19}}{d_{16}}+d_{20} e^{d_{16} v / 2}
\end{aligned}
$$

To obtain the solution of equation (175) we insert equation (176) and (177) in equation (174) and we get

$$
r^{2} C_{3}^{\prime \prime}+r C_{3}^{\prime}=2 d_{19} d_{17} r^{d_{16}}
$$

Solution of equation (179) is

$$
C_{3}(r)=\frac{2 d_{17} d_{19}}{d_{16}^{2}} r^{d_{16}}+d_{21} \ln r+d_{22}
$$

Substituting equation (177-178) and equation (180) in equation (168), we get

$$
A=d_{22}-\frac{2 d_{18} d_{19}}{d_{16}}+d_{21} \ln r+S_{5}(v)+d_{18} d_{20} e^{d_{16} v / 2}+\frac{2 d_{17} d_{19}}{d_{16}^{2}} r^{d_{16}}+\frac{d_{17}}{d_{16}} r^{d_{16}}\left[d_{20} e^{d_{16} v / 2}-\frac{2 d_{19}}{d_{16}}\right]+e^{-2 v}\left\{\frac{R_{e}}{c_{4}^{2}}\left(\frac{1}{r^{2}}\right)-\frac{4 d_{14}}{r^{4}}+d_{15}\right\}
$$

The viscosity is obtained from equation (167) by substituting equation (114) and equation (181) which is

$$
\begin{aligned}
\mu(r, v)=\left(\frac{-r^{2} e^{-v}}{2 c_{4}}\right) & {\left[d_{22}-\frac{2 d_{18} d_{19}}{d_{16}}+d_{21} \ln r+S_{5}(v)+d_{18} d_{20} e^{d_{16} / 2}+\frac{2 d_{17} d_{19}}{d_{16}^{2}} r^{d_{16}}+\frac{d_{17}}{d_{16}} r^{d_{16}}\left[d_{20} e^{d_{16} v / 2}-\frac{2 d_{19}}{d_{16}}\right]\right.} \\
& \left.+e^{-2 v}\left\{\frac{R_{e}}{c_{4}^{2}}\left(\frac{1}{r^{2}}\right)-\frac{4 d_{14}}{r^{4}}+d_{15}\right\}\right]
\end{aligned}
$$

The solution of equations (61) and (62), utilizing equations (165) and equation (181), is 
$R_{e} L=R_{e}\left(\frac{1}{r^{2} c_{4}{ }^{2}} e^{-2 v}\right)+r\left[C_{3}^{\prime}(r) v+C_{4}^{\prime}(r) \int S_{6}(v) d v+\left(\frac{e^{-2 v}}{-2}\right) D_{1}^{\prime}(r)\right]-A+2 d_{21} \ln r+2 d_{22}-\frac{4 d_{18} d_{19}}{d_{16}}+p_{3}$

where $p_{3}$ is constant.

The equation (64) of temperature distribution, on substituting equations (165) and equation (181), becomes

$$
\begin{gathered}
r^{2} T_{r r}-2 r T_{v r}+\frac{2}{r} T_{v v}\left(v^{\prime}\right)+r\left(1-\frac{R_{e} P_{r}}{c_{4}} e^{-v}\right) T_{r} \\
=\frac{2 E_{c} P_{r} d_{21}}{c_{4}} \ln r e^{-v}+\frac{2 E_{c} P_{r} d_{22}}{c_{4}} e^{-v}+\frac{2 E_{c} P_{r}}{c_{4}} S_{5}(v) e^{-v}-\frac{4 E_{c} P_{r} d_{19} d_{18}}{c_{4} d_{16}} e^{-v}+\frac{2 E_{c} P_{r} d_{18} d_{20}}{c_{4}} e^{\left(\frac{d_{16}}{2}-1\right) v}+\frac{2 E_{c} P_{r} d_{17} d_{20}}{c_{4} d_{16}} r^{d_{16}} e^{\left(\frac{d_{16}}{2}-1\right) v} \\
\quad+\frac{2 E_{c} P_{r}}{c_{4}} e^{-3 v}\left\{\frac{R_{e}}{c_{4}{ }^{2}}\left(\frac{1}{r^{2}}\right)-\frac{4 d_{14}}{r^{4}}+d_{15}\right\}
\end{gathered}
$$

It is obvious from equation (184) the general solution is extremely difficult to obtain, however the terms in equation (184) suggests to seek a solution of the form

$T=e^{-v} T_{2}(r)+S_{7}(v)+r^{b} S_{8}(v)$

On substituting equation (185) in equation (184) we get

$$
\begin{aligned}
& e^{-v}\left[r^{2} T_{2}^{\prime \prime}(r)+3 r T_{2}^{\prime}(r)+2 T_{2}(r)\right]+r^{b}\left[2 S_{8}^{\prime \prime}(v)-2 b S_{8}^{\prime}(v)+b^{2} S_{8}(v)-\frac{R_{e} P_{r}}{c_{4}} e^{-v} b S_{8}(v)\right]+2 S_{7}^{\prime \prime}(v)-\frac{R_{e} P_{r}}{c_{4}} e^{-2 v} r T_{2}^{\prime}(r) \\
& =e^{-v}\left[\frac{2 E_{c} P_{r} d_{21}}{c_{4}} \ln r\right]+e^{-v}\left[\frac{2 E_{c} P_{r}}{c_{4}}\left(d_{22}-\frac{2 d_{19} d_{18}}{d_{16}}\right)+\frac{2 E_{c} P_{r}}{c_{4}} S_{5}(v)+\frac{2 E_{c} P_{r} d_{18} d_{20}}{c_{4}} e^{\left(\frac{d_{16}}{2}-1\right) v}\right] \\
& +\frac{2 E_{c} P_{r} d_{17} d_{20}}{c_{4} d_{16}} r^{d_{16}} e^{\left(\frac{d_{16}}{2}-1\right) v}+\frac{2 E_{c} P_{r}}{c_{4}} e^{-3 v}\left[\frac{R_{e}}{c_{4}^{2}}\left(\frac{1}{r^{2}}\right)\right]+\frac{2 E_{c} P_{r}}{c_{4}} e^{-3 v}\left[-\frac{4 d_{14}}{r^{4}}+d_{15}\right]
\end{aligned}
$$

On comparing the right-hand side and left-hand side, we get

$$
\begin{aligned}
& T_{2}(r)=\frac{E_{c} d_{17} d_{20}}{R_{e}}\left(\frac{r^{-2}}{-2}\right) \\
& 2 S_{7}^{\prime \prime}(v)=\frac{2 E_{c} P_{r} d_{22}}{c_{4}} e^{-v}+\frac{2 E_{c} P_{r}}{c_{4}} e^{-v} S_{5}(v)-\frac{4 E_{c} P_{r} d_{19} d_{18}}{c_{4} d_{16}} e^{-v}+\frac{2 E_{c} P_{r} d_{18} d_{20}}{c_{4}} e^{\left(\frac{d_{16}}{2}-1\right) v}
\end{aligned}
$$

and

$$
S_{8}^{\prime \prime}(v)+2 S_{8}^{\prime}(v)+\left(2+\frac{R_{e} P_{r}}{c_{4}} e^{-v}\right) S_{8}(v)=\frac{E_{c} d_{17} d_{20}}{2 R_{e}} e^{-v}+\frac{E_{c} R_{e} P_{r}}{c_{4}{ }^{3}} e^{-3 v}
$$

provided

$$
d_{14}=0, \quad d_{15}=0 \quad d_{16}=-2 \quad d_{21}=0, \quad b=-2
$$

Solution of equation (188) is

$$
S_{7}(v)=\left[\frac{E_{c} P_{r} d_{22}}{c_{4}}-\frac{2 E_{c} P_{r} d_{10} d_{18}}{c_{4} d_{16}}\right] e^{-v}+\frac{E_{c} P_{r}}{c_{4}} \iint e^{-v} S_{5}(v) d v+\frac{E_{c} P_{r} d_{18} d_{20}}{2 c_{4}} e^{-2 v}+s_{3} v+s_{4}
$$

The solution of equation (189) is 


$$
\begin{aligned}
S_{8}(v)=C_{3} & \left.A 5 e^{-v} \text { BesselJ }\left[-2 i, 2 \sqrt{A 5 e^{-v}}\right] \text { Gamma[1-2i }\right] \\
& \left.+C_{4} A 5 e^{-v} \text { BesselJ }\left[2 i, 2 \sqrt{A 5 e^{-v}}\right] \text { Gamma[1+2i }\right] \\
& \left.+\frac{i}{2} e^{-v} \text { Gamma[1-2i]Gamma[1+2i }\right] \\
& \left\{\text { BesselJ }\left[2 i, 2 \sqrt{A 5 e^{-v}}\right] \int e^{-v}\left(A 7+A 6 e^{2 v}\right) \text { BesselJ }\left[-2 i, 2 \sqrt{A 5 e^{-v}}\right] d v\right. \\
& \left.- \text { BesselJ }\left[-2 i, 2 \sqrt{A 5 e^{-v}}\right] \int e^{-v}\left(A 7+A 6 e^{2 v}\right) \operatorname{BesselJ}\left[2 i, 2 \sqrt{A 5 e^{-v}}\right] d v\right\}
\end{aligned}
$$

where $C_{3}$ and $C_{4}$ are constant and

$$
A 5=\frac{R_{e} P_{r}}{c_{4}}, \quad A 6=\frac{E_{c} d_{17} d_{20}}{2 R_{e}}, \quad A 7=\frac{E_{c} P_{r} R_{e}}{c_{4}{ }^{3}}
$$

Case II:

For this case $g(r) \neq 1$ and basic flow equations are

$$
\begin{aligned}
& q=\frac{\sqrt{1+(M+N v)^{2}}}{r g v^{\prime}} \\
& w=\left[\frac{f^{\prime}(r)}{r g(r)}+\frac{f^{\prime \prime}(r)}{g(r)}-\frac{2 f^{\prime}(r) g^{\prime}(r)}{g^{2}(r)}\right]\left(\frac{1}{v^{\prime}(\psi)}\right)+\left[\frac{g^{\prime}(r)}{r g(r)}+\frac{g^{\prime \prime}(r)}{g(r)}-\frac{2\left\{g^{\prime}(r)\right\}^{2}}{g^{2}(r)}\right]\left(\frac{v(\psi)}{v^{\prime}(\psi)}\right) \\
& +\left[\frac{1}{r^{2} g^{2}(r)}+\frac{\left\{f^{\prime}(r)\right\}^{2}}{g^{2}(r)}\right]\left(\frac{v^{\prime \prime}(\psi)}{\left\{v^{\prime}(\psi)\right\}^{3}}\right)+\frac{2 f^{\prime}(r) g^{\prime}(r) v(\psi) v^{\prime \prime}(\psi)}{g^{2}(r)\left\{v^{\prime}(\psi)\right\}^{3}}+\frac{\left\{g^{\prime}(r)\right\}^{2} v^{2}(\psi) v^{\prime \prime}(\psi)}{g^{2}(r)\left\{v^{\prime}(\psi)\right\}^{3}} \\
& -R_{e} w=R_{e} v^{\prime} L_{v}-\left(r g v^{\prime}\right) A_{r}+(M+N v) v^{\prime} A_{v}+v^{\prime} B_{v} \\
& 0=-R_{e} L_{r}+\frac{A_{v}(2-E)}{(r g)}+A_{r}(M+N v)-\frac{(M+N v) B_{v}}{(r g)} \\
& r g v^{\prime} A_{r r}-2(M+N v) v^{\prime} A_{r v}-\frac{\left[1-(M+N v)^{2}\right]}{r g v^{\prime}}\left(v^{\prime \prime} A_{v}+v^{\prime 2} A_{v v}\right) \\
& +g v^{\prime} A_{r}-v^{\prime} A_{v}\left(M^{\prime}+N^{\prime} v\right)-v^{\prime}\left\{B_{r}-\frac{\left(f^{\prime}+g^{\prime} v\right) B_{v}}{g}\right\}_{v}=R_{e} w_{r}
\end{aligned}
$$

$$
\begin{gathered}
\left(r g v^{\prime}\right) T_{r r}-2(M+N v) T_{v r} v^{\prime}+\frac{\left[1+(M+N v)^{2}\right]}{\left(r g v^{\prime}\right)} T_{v v}\left(v^{\prime}\right)^{2}+\left(\left(r g v^{\prime}\right)_{r}-\frac{\left[1+(M+N v)^{2}\right]}{2(M+N v)}-R_{e} P_{r}\right) T_{r} \\
+\left[\frac{[2 N(M+N v)]}{(r g)}-\left(M^{\prime}+N^{\prime} v\right)-\frac{\left[1+(M+N v)^{2}\right]\left(r g v^{\prime \prime}\right)}{\left(r g v^{\prime}\right)^{2}}+\frac{\left[1+(M+N v)^{2}\right]}{\left(r g v^{\prime}\right)}\left(\frac{v^{\prime \prime}}{v^{\prime}}\right)\right] T_{v} v^{\prime} \\
=-\frac{\left(r g v^{\prime}\right) E_{c} P_{r}}{4 \mu}\left(B^{2}+4 A^{2}\right)
\end{gathered}
$$

where

$$
\begin{aligned}
& A(r, \psi)=\frac{\mu}{\left(r g v^{\prime}\right)}\left[\frac{-2(r g)_{r}(M+N v)}{(r g)}+\left(M^{\prime}+N^{\prime} v\right)-\frac{\left\{\left(1-(M+N v)^{2}\right\} v^{\prime \prime}\right.}{r g v^{\prime 2}}\right] \\
& B(r, \psi)=\frac{4 \mu}{\left(r g v^{\prime}\right)^{3}}\left[-v^{\prime 2}(r g)(r g)_{r}+(M+N v)\left(r g v^{\prime \prime}\right)\right] \\
& M(r)=r f^{\prime}(r) \quad \text { and } \quad N(r)=r g^{\prime}(r)
\end{aligned}
$$

The above system of equations indicates that its solutions strongly depend on the function $v(\psi)$ and its derivatives. Since $v^{\prime}(\psi) \neq 0$, following the Case I, we consider the following cases

Case II(a) $\quad v^{\prime \prime}(\psi)=0$ or $v^{\prime}(\psi)=a$ 
Case II(b) $\quad v^{\prime \prime}(\psi) \neq 0$

Case II(a):

For this case the equations(197-204) becomes

$$
\begin{aligned}
& q=\frac{\sqrt{1+(M+N v)^{2}}}{\arg } \\
& w=\left[\frac{f^{\prime}(r)}{r g(r)}+\frac{f^{\prime \prime}(r)}{g(r)}-\frac{2 f^{\prime}(r) g^{\prime}(r)}{g^{2}(r)}\right]\left(\frac{1}{a}\right)+\left[\frac{g^{\prime}(r)}{r g(r)}+\frac{g^{\prime \prime}(r)}{g(r)}-\frac{2\left\{g^{\prime}(r)\right\}^{2}}{g^{2}(r)}\right]\left(\frac{v(\psi)}{a}\right) \\
& -R_{e} w=a R_{e} L_{v}-(\arg ) A_{r}+a(M+N v) A_{v}+a B_{v} \\
& 0=-R_{e} L_{r}+\frac{A_{v}(2-E)}{(r g)}+A_{r}(M+N v)-\frac{(M+N v) B_{v}}{(r g)} \\
& \arg A_{r r}-2 a(M+N v) A_{r v}-\frac{a\left[1-(M+N v)^{2}\right]}{r g} A_{v v}+a g A_{r}-a\left(M^{\prime}+N^{\prime} v\right) A_{v}-a\left\{B_{r}-\frac{\left(f^{\prime}+g^{\prime} v\right) B_{v}}{g}\right\}_{v}=R_{e} w_{r} \\
& (\arg ) T_{r r}-2 a(M+N v) T_{v r}+\frac{a\left[1+(M+N v)^{2}\right]}{r g} T_{v v}+\left((a r g)_{r}-\frac{a\left[1+(M+N v)^{2}\right]}{2(M+N v)}-R_{e} P_{r}\right) T_{r} \\
& \left.+a\left[\frac{[2 N(M+N v)]}{(r g)}-\left(M^{\prime}+N^{\prime} v\right)\right]\right] T_{v}=-\frac{(a r g) E_{c} P_{r}}{4 \mu}\left(B^{2}+4 A^{2}\right)
\end{aligned}
$$$$
A(r, \psi)=\frac{\mu}{(\arg )}\left[\left\{\frac{-2(r g)_{r} M}{(r g)}+M^{\prime}\right\}-v\left\{\frac{2(r g)_{r} N}{(r g)}-N^{\prime}\right\}\right]
$$$$
B(r, \psi)=\frac{-4 a \mu}{(a r g)^{2}}(r g)_{r}
$$

It is obvious from equation (207), it is extremely difficult to obtain its exact solutions. However, we see that by setting $A=0$ or $B=0$ we can reduce the equation (207) to simple whose solutions are determinable.

For

$A=0$

On substituting equations (202) and (209) in equation (211) and comparing the coefficient of $v$, we get

$$
\begin{aligned}
& \frac{2(r g)_{r} g^{\prime}}{g}-\left(r g^{\prime}\right)^{\prime}=0 \\
& \frac{-2(r g)_{r} f^{\prime}}{g}+\left(r f^{\prime}\right)^{\prime}=0
\end{aligned}
$$

The solution of equation (212) is

$$
g(r)=\frac{-1}{\left(C_{0} r^{2}+C_{1}\right)}
$$

Utilizing equation (215) in equation (213) we get

$$
r\left(C_{0} r^{2}+C_{1}\right) f^{\prime \prime}+\left(3 C_{0} r^{2}-C_{1}\right) f^{\prime}=0
$$

The equation (215) possesses trivial and non-trivial solutions. For trivial solution 
$f(r)=0$

The equation (209), becomes

$B_{r v}-\left(\frac{g^{\prime}}{g}\right) v B_{v v}-\left(\frac{g^{\prime}}{g}\right) B_{v}=\frac{-4 R_{e} C_{0} v}{a^{2}} g^{\prime}$

This suggests to seek a solution of the form

$B=v^{2} Q(r)$

On substituting equation (218) in equation (217) we get

$Q^{\prime}-2\left(\frac{g^{\prime}}{g}\right) Q=\frac{-2 R_{e} C_{0}}{a^{2}} g^{\prime}$

whose solution is

$Q(r)=\frac{2 R_{e} C_{0}}{a^{2}} g+C_{2} g^{2}$

On substituting equation (220) in equation (218) we get

$B=v^{2}\left\{\frac{2 R_{e} C_{0}}{a^{2}} g+C_{2} g^{2}\right\}$

Equation (204) gives the value of $\mu$

$\mu=\frac{-a(r g)^{2}}{4(r g)^{\prime}}\left\{\frac{2 R_{e} C_{0}}{a^{2}} g+C_{2} g^{2}\right\} v^{2}$

Solution of equations (197) and (198) utilizing equation (212) and (213) is

$a R_{e} L=\left\{-\frac{R_{e}}{a}\left[\frac{N^{\prime}}{r g}-\frac{2 N^{2}}{(r g)^{2}}\right]-2 a Q(r)\right\}\left(\frac{v^{2}}{2}\right)+p_{6}$

The energy equation for this is

$$
\begin{aligned}
(a r g) T_{r r}-2 a\left(r g^{\prime} v\right) T_{v r}+\frac{a\left[1+\left(r g^{\prime} v\right)^{2}\right]}{r g} T_{v v} & +\left((a r g)_{r}-\frac{a\left[1+\left(r g^{\prime} v\right)^{2}\right]}{2\left(r g^{\prime} v\right)}-R_{e} P_{r}\right) T_{r} \\
& +a\left[\frac{\left[2 r g^{\prime}\left(r g^{\prime} v\right)\right]}{(r g)}-\left(r g^{\prime}\right)^{\prime} v\right] T_{v}=E_{c} P_{r} a^{2}(r g)\left(r g^{\prime}+g\right) v^{2} Q(r)
\end{aligned}
$$

We seek a solution of equation (224) of the form

$T(r, v)=v^{2} R(r)+S(r)$

Inserting equation (225) in (224) we find

$$
\begin{aligned}
& a r^{2} g^{2} R^{\prime \prime}+r g\left\{a g-R_{e} P_{r}-4 a r g^{\prime}\right\} R^{\prime}+2 a\left\{3\left(r g^{\prime}\right)^{2}-r g\left(r g^{\prime}\right)^{\prime}\right\} R=E_{c} P_{r}\left(r g^{\prime}+g\right)\left\{\frac{2 R_{e} C_{0}}{a^{2}} g+C_{2} g^{2}\right\} \\
& a r^{2} g^{2} S^{\prime \prime}+r g\left\{a g-R_{e} P_{r}\right\} S^{\prime}=-2 a R(r)
\end{aligned}
$$

On substituting the value of $g(r)$ from equation (214) in equation (226) we get 


$$
\begin{aligned}
a r^{2}\left(C_{0} r^{2}+C_{1}\right)^{2} R^{\prime \prime}+r\left(C_{0} r^{2}+C_{1}\right)\left\{a\left(9 C_{0} r^{2}+C_{1}\right)+R_{e} P_{r}\left(C_{0} r^{2}+C_{1}\right)^{2}\right\} R^{\prime}+8 a C_{0} r^{2}\left(2 C_{0} r^{2}+C_{1}\right) R \\
=-E_{c} P_{r}\left(C_{0} r^{2}+C_{1}\right)\left\{\frac{2 R_{e} C_{0}^{2}}{a^{2}} r^{2}+\frac{2 R_{e} C_{0} C_{1}}{a^{2}}-C_{2}\right\}
\end{aligned}
$$

where $C_{0}, C_{1}$ and $C_{2}$ are arbitrary constants. Equation (228) suggests that a solution can be determined by setting

$$
C_{1}=0
$$

Equation (228) on substituting $C_{1}=0$, becomes

$$
a r^{2} R^{\prime \prime}+r\left\{9 a+R_{e} P_{r} C_{0} r^{2}\right\} R^{\prime}-16 a R=-E_{c} P_{r}\left\{\frac{2 R_{e} C_{0}^{2}}{a^{2}}-\frac{C_{2}}{C_{0} r^{2}}\right\}
$$

The equation suggest to seek a solution of the form

$$
R(r)=B_{1}+B_{2} r^{-2}
$$

On substituting equation (231) in (230) we get

$$
B_{1}=\frac{E_{c} P_{r} R_{e}}{8 a^{2}}\left(\frac{1}{a}+\frac{P_{r} C_{2}}{28}\right)
$$

and

$$
B_{2}=\frac{-E_{c} P_{r} C_{2}}{28 a C_{0}}
$$

Inserting equation (232) and (233) in equation (231) we get

$$
R(r)=\frac{E_{c} P_{r} R_{e}}{8 a^{2}}\left(\frac{1}{a}+\frac{P_{r} C_{2}}{28}\right)-\frac{E_{c} P_{r} C_{2}}{28 a C_{0}} r^{-2}
$$

Employing equation (234) in equation (227) we get

$$
S(r)=-2 C_{0}^{2} \int\left[\frac{e^{\frac{-C_{0} R_{0} P_{P_{t}}}{2 a} r^{2}}}{r} \int r^{3} e^{\frac{C_{0} R_{2} P_{r}}{2 a} r^{2}} R(r) d r\right] d r+C_{3} \int \frac{e^{\frac{-C_{0} R_{r} P_{r}}{2 a} r^{2}}}{r} d r+C_{4}
$$

On substituting equation (234) and (235) in equation (225) we get the temperature distribution. The above solutions are when the function $A=0$.

Now when the function $B$ is zero equation (208) gives

$$
g=\frac{c}{r}
$$

where $c$ is a non-zero constant.

Inserting equation (236) in equation (205) we get

$$
\begin{aligned}
a_{1} c A_{r r}- & 2 a(M-g v) A_{r v}-\frac{a\left[1-(M-g v)^{2}\right]}{c} A_{v v}+a g A_{r}+a A_{v}\left(-\left(M^{\prime}-g^{\prime} v\right)+\frac{2(-g)(M-g v)}{c}\right) \\
= & \left(\frac{R_{e}}{a c}\right)\left[M^{\prime}+\frac{2 M}{r}\right]^{\prime}+\frac{2 R_{e} v}{a r^{3}}
\end{aligned}
$$

The right hand side suggests seeking a solution of the form

$$
A(r, \psi)=R(r)+P(r) v
$$


Equation (237) employing equation (238) become

$a c R^{\prime \prime}-2 a M P^{\prime}+a g R^{\prime}+a P\left(-M^{\prime}-\frac{2 g M}{c}\right)+v\left\{a c P^{\prime \prime}+3 a g P^{\prime}+a P\left(g^{\prime}+\frac{2 g^{2}}{c}\right)\right\}=\left(\frac{R_{e}}{a c}\right)\left[M^{\prime}+\frac{2 M}{r}\right]^{\prime}+\frac{2 R_{e} v}{a r^{3}}$

Since $v$ and $r$ independent variables, equation (239) yields

$r^{2} P^{\prime \prime}+3 r P^{\prime}+P=-\frac{2 R_{e}}{a^{2} c r}$

and

$\left(r R^{\prime}\right)^{\prime}=\left(\frac{r R_{e}}{a^{2} c^{2}}\right)\left[M^{\prime}+\frac{2 M}{r}\right]^{\prime}+\frac{2 r M}{c} P^{\prime}+\frac{r}{c}\left(M^{\prime}+\frac{2 M}{r}\right) P$

The solution of equations (240) and (241) are

$P(r)=\frac{s_{1}}{r}+\frac{s_{2} \ln r}{r}-\frac{R_{e}(\ln r)^{2}}{a^{2} c r}$

and

$R(r)=\frac{1}{r} \int\left\{\frac{1}{r} \int Z_{1}(r) d r\right\} d r+C_{1} \ln r+C_{2}$

where

$Z_{1}(r)=\left(\frac{r R_{e}}{a^{2} c^{2}}\right)\left[M^{\prime}+\frac{2 M}{r}\right]^{\prime}+\frac{2 r M}{c} P^{\prime}+\frac{r}{c}\left(M^{\prime}+\frac{2 M}{r}\right) P$

On substituting equation (238) in equation (207) we get the value of $\mu$ which is

$\mu=\frac{a c}{\left(M^{\prime}-g^{\prime} v\right)}(R(r)+P(r) v)$

where the function $R(r)$ and $P(r)$ are given by equations (243) and (232) respectively.

The solution of equation (203) and (204) using equation (236), is

$$
\begin{aligned}
a R_{e} L= & \left(\frac{-R_{e}}{a r^{2}}+a c P^{\prime}(r)+a g P(r)\right)\left(\frac{v^{2}}{2}\right)+\left\{\left(\frac{-R_{e}}{a c}\right)\left[M^{\prime}(r)+\frac{2 M(r)}{r}\right]\right. \\
& \left.+a c R^{\prime}(r)-a M(r) P(r)\right\} v+\frac{a}{c} \int\left(1-M(r)^{2}\right) P(r) d r+\int M(r) R^{\prime}(r) d r+p_{5}
\end{aligned}
$$

Which gives the generalized energy function $L$ for the case when $B=0$.

The equation (206) for temperature distribution utilizing equation (236) becomes

$$
\begin{gathered}
a c T_{r r}-2 a(M-g v) T_{v r}+\frac{a\left[1+(M-g v)^{2}\right]}{c} T_{v v}+\left(\frac{a c}{r}-R_{e} P_{r}\right) T_{r}+\left(-\frac{2(M+N v)}{r}-\left(M^{\prime}-g^{\prime} v\right)\right) a T_{v} \\
=-E_{c} P_{r}\left[R M^{\prime}+\left(P M^{\prime}-g^{\prime} R\right) v-P g^{\prime} v^{2}\right]
\end{gathered}
$$

The right-hand side of equation (247) suggests to seek solution of the form

$T(r, \psi)=R_{1}(r)+R_{2}(r) v+R_{3}(r) v^{2}$

Inserting equation (248) in equation (247) we get 
$v^{2}\left[a c R_{3}^{\prime \prime}+4 a g R_{3}^{\prime}+\frac{2 a R_{3} g^{2}}{c}+2 a R_{3}\left(g^{\prime}+\frac{2 g}{r}\right)+\left(\frac{a c}{r}-R_{e} P_{r}\right) R_{3}^{\prime}\right]+v\left[a c R_{2}^{\prime \prime}-4 a M R_{3}^{\prime} v+2 a g v R_{2}^{\prime}-\frac{4 a R_{3} M g v}{c}\right.$

$$
\begin{aligned}
+\left(\frac{a c}{r}-R_{e} P_{r}\right) R_{2}^{\prime} v+ & \left.a\left\{-2 R_{3}\left(M^{\prime}+\frac{2 M}{r}\right)+R_{2}\left(g^{\prime}+\frac{2 g}{r}\right)\right\} v\right] \\
+a c R_{1}^{\prime \prime} & -2 a M R_{2}^{\prime}+\frac{2 a R_{3}\left(1+M^{2}\right)}{c}+\left(\frac{a c}{r}-R_{e} P_{r}\right) R_{1}^{\prime}+\left(-a\left(M^{\prime}+\frac{2 M}{r}\right) R_{2}\right) \\
& =-E_{c} P_{r}\left[R M^{\prime}+\left(P M^{\prime}-g^{\prime} R\right) v-P g^{\prime} v^{2}\right]
\end{aligned}
$$

Equation (249) on comparing the coefficients of $v^{0}, v^{1}$ and $v^{2}$ we get

$$
\begin{aligned}
& r^{2} R_{3}^{\prime \prime}+\left(5 r-\frac{R_{e} P_{r}}{a c} r^{2}\right) R_{3}^{\prime}+4 R_{3}=Z_{4}(r) \\
& r^{2} R_{2}^{\prime \prime}+\left(3 r-\frac{R_{e} P_{r} r^{2}}{a c}\right) R_{2}^{\prime}+R_{2}=Z_{3}(r)
\end{aligned}
$$

and

$$
R_{1}^{\prime \prime}+\left(\frac{1}{r}-\frac{R_{e} P_{r}}{a c}\right) R_{1}^{\prime}=Z_{2}(r)
$$

where

$$
\begin{aligned}
& Z_{2}(r)=\frac{2 M R_{2}^{\prime}}{c}+\frac{1}{c}\left(M^{\prime}+\frac{2 M}{r}\right) R_{2}-\left(\frac{2\left(1+M^{2}\right)}{c^{2}}\right) R_{3}-\frac{E_{c} P_{r} M R}{a c} R \\
& Z_{3}(r)=4 a r^{2} M R_{3}^{\prime}+2 a r^{2} R_{3}\left(M^{\prime}+\frac{4 M}{r}\right)-E_{c} P_{r}\left(r^{2} P^{\prime} M^{\prime}+c R\right) \\
& Z_{4}(r)=\frac{-E_{c} P_{r}}{a} P(r)
\end{aligned}
$$

The solution of equation (252) is

$$
R_{1}(r)=\left\{\left\{\frac{e^{\left(\frac{R_{e} P_{r}}{a c}\right) r}}{r} \int r e^{-\left(\frac{R_{e} P_{r}}{a c}\right) r} Z_{2}(r) d r\right\} d r+C_{5} \int\left\{\frac{e^{\left(\frac{R_{e} P_{r}}{a c}\right) r}}{r}\right\} d r+C_{6}\right.
$$

The solutions of equations (250) and (251) using Mathematica is

$$
\begin{aligned}
& R_{2}(r)=C_{3}\left(-1+\frac{1}{A 1 r}\right)+C_{4} \text { MeijerG }[\{\{\},\{1\}\},\{\{-1,-1\},\{\}\},-\mathrm{A} 1 \mathrm{r}] \\
& +\frac{1}{r}\left\{A 1(-1+A 1 r) \int e^{-A 1 r} r \text { MeijerG[ }[\{\{\},\{1\}\},\{\{-1,-1\},\{\}\},-\mathrm{A} 1 \mathrm{r}] Z_{3}(r) d r\right. \\
& \left.+r \text { MeijerG }[\{\{\},\{1\}\},\{\{-1,-1\},\{\}\},-\mathrm{A} 1 \mathrm{r}] \int A 1(1-A 1 r) e^{-A 1 r} Z_{3}(r) d r\right\} \\
& R_{3}(r)=C_{1}\left(\frac{1}{2}-\frac{2}{A 1 r}+\frac{1}{(A 1 r)^{2}}\right) \\
& +C_{2} \text { MeijerG }[\{\{\},\{1\}\},\{\{-2,-2\},\{\}\},-\mathrm{A} 1 \mathrm{r}] \\
& +\frac{1}{r^{2}}\left\{A 1^{2}\left(2-4 A 1 r+A 1^{2} r^{2}\right) \int e^{-A 1 r} r^{3} \text { MeijerG }[\{\{\},\{1\}\},\{\{-2,-2\},\{\}\},-\mathrm{A} 1 \mathrm{r}] Z_{4}(r) d r\right. \\
& \left.+r^{2} \text { MeijerG[\{\{\},\{1\}\},\{\{-2,-2\},\{\}\},-A1 r] } \int A 1^{2}\left(-2+4 A 1 r-A 1^{2} r^{2}\right) r e^{-A 1 r} Z_{4}(r) d r\right\}
\end{aligned}
$$

where 
$A 1=\frac{R_{e} P_{r}}{a c}$

Using $R_{1}(r), R_{2}(r)$ and $R_{3}(r)$ in equation (248) we get temperature distribution.

Case II(b):

When $g(r) \neq 1$ and $v^{\prime \prime}(\psi) \neq 0$ the system of equations (195-200) is extremely difficult to solve in general. However the system simplifies into a very simple system if we assume

$v(\psi)=e^{\psi}$

On utilizing equation (260) in equations (198) and (199) we get

$A=\frac{\mu}{r^{2} g^{2} v^{2}}\left[v^{2}\left[r g N^{\prime}+N^{2}-2 N(N+g)\right]+v\left[r g M^{\prime}-2 M g\right]-\left(1-M^{2}\right)\right]$

$B=\frac{4 \mu}{r^{2} g^{2} v^{2}}[(M-g v)]$

where

$M(r)=r f^{\prime}(r) \quad$ and $\quad N(r)=r g^{\prime}(r)$

On eliminating $\mu$ from equations (262) and (263) we get

$\frac{4 A}{B}=-\frac{R_{2}}{g} v+\frac{\left(R_{1}+\frac{M R_{2}}{g}\right)+R_{0}}{(M-g v)}$

where

$R_{2}(r)=r g N^{\prime}+N^{2}-2 N(N+g)$

$R_{1}(r)=r g M^{\prime}-2 M g$

$R_{0}(r)=-\left(1-M^{2}\right)$

On setting

$R_{0}=0$

and

$\left(R_{1}+\frac{M R_{2}}{g}\right)=0$

We can further simplify equation (264) leading to achieve exact solution. Equation $R_{0}=0$, provides

$M= \pm 1$.

When $M=1$, we found that the equation (196) admits exact solution. For $M=-1$, the temperature distribution equation is not exactly solvable, therefore this case is discarded.

$r f^{\prime}(r)=1$

whose solution is

$f(r)=\ln r+m_{1}$ 
where $m_{1}$ is constant.

Now equation (269) utilizing equation (266) and (267) becomes

$r^{2} K^{\prime}-r K-2=0$

where

$K=\left(\frac{g^{\prime}}{g}\right)$

The equation (273) admits two solutions. One is particular and another is general. The particular and general solutions are

$g=\frac{c}{r}$

and

$g=r e^{\frac{1}{2} r^{2} m_{2}+m_{3}}$

where $m_{2}, m_{3}$ are constants

Equation (264) employing equation (260) and (269) becomes

$B=\frac{-2 r}{c e^{\psi}} A$

Inserting equations (274), (275) and (277) in equation (199) we get

$c v A_{r r}+\left(-2 v+\frac{2 c v^{2}}{r}+\frac{2 r}{c}\right) A_{r v}+\left(-\frac{2 r}{c^{2}}+\frac{2 v}{c}-\frac{2 v^{2}}{r}+\frac{c v^{3}}{r^{2}}\right) A_{v v}+\left(\frac{c v}{r}-\frac{2 r}{c v}\right) A_{r}+\left(-\frac{2 v}{r}+\frac{c v^{2}}{r^{2}}+\frac{4 r}{c^{2} v}\right) A_{v}-\frac{4 r}{c^{2} v^{2}} A=0$

The variable coefficients of equation (278) suggest to seek solution of the form

$A(r, \psi)=D r^{n} v^{m}$

where $D$ is constant.

Utilizing equation (279) in (278) we find

$$
\begin{aligned}
D r^{n} v^{m}[ & \frac{v}{r^{2}}\{c n(n-1)+2 c n m+c m(m-1)+n c+c m\}+\frac{1}{v}\left\{\frac{2 n m}{c}+\frac{2 m(m-1)}{c}-\frac{2 n}{c}\right\} \\
\left.+\frac{1}{r}\{-2 n m-2 m(m-1)-2 m\}+\frac{r}{v^{2}}\left\{-\frac{2 m(m-1)}{c^{2}}+\frac{4 m}{c^{2}}-\frac{4}{c^{2}}\right\}\right] & =0
\end{aligned}
$$

Equation (280) is identically satisfied provided

$$
\begin{aligned}
& n(n-1)+2 n m+m(m-1)+n+m=0 \\
& n m+m(m-1)-n=0 \\
& n m+m(m-1)+m=0 \\
& m(m-1)-2 m+2=0
\end{aligned}
$$

The solution of equations (281-284) is 
$m=1,2$

and

$n=-m$

For $(m, n)=(1,-1)$ the equation (262) and (279) gives

$\mu=\left(\frac{-D c}{2}\right)\left(\frac{r v^{2}}{(r-c v)}\right)$

and

$A=D\left(\frac{v}{r}\right)$

The solution of equation (194) and (195) when $(m, n)=(1,-1)$ is

$R_{e} L=\left(\frac{R_{e}}{c^{2} v^{2}}\right)-D\left(\frac{v}{r}\right)+p_{7}$

where $p_{7}$ is constant.

The energy equation is

$$
\begin{aligned}
(c v)^{2} T_{r r} & -2\left(1-\frac{c v}{r}\right) c v^{2} T_{v r}+v^{2}\left\{2-\frac{2 c v}{r}+\frac{c^{2} v^{2}}{r^{2}}\right\} T_{v v}+\left(\frac{c^{2} v^{2}}{r}-c v R_{e} P_{r}\right) T_{r}+\left(-\frac{2 c v^{2}}{r}+\frac{c^{2} v^{3}}{r^{2}}\right) T_{v} \\
& =\frac{2 D E_{c} P_{r}}{c}\left[1-\left(\frac{c v}{r}\right)+\left(\frac{c v}{r}\right)^{2}-\left(\frac{c v}{r}\right)^{3}\right]
\end{aligned}
$$

In the light of the coefficient of equation (288) we seek solution of the form

$$
T=a \ln r+b \ln v+T_{0}+T_{1}\left(\frac{v}{r}\right)+T_{2}\left(\frac{v}{r}\right)^{2}
$$

Inserting equation (289) in equation (288) we get

$$
\begin{aligned}
& \left(\frac{v}{r}\right)^{4}\left\{c^{2}\left(6 T_{2}\right)+2 c^{2}\left(-4 T_{2}\right)+c^{2}\left(2 T_{2}\right)+c^{2}\left(-2 T_{2}\right)+c^{2}\left(2 T_{2}\right)\right\} \\
& +\left(\frac{v}{r}\right)^{3}\left\{\begin{array}{c}
c^{2}\left(2 T_{1}\right)+2 c^{2}\left(-T_{1}\right)-2 c\left(-4 T_{2}\right)-2 c\left(2 T_{2}\right) \\
+c^{2}\left(-T_{1}\right)-c R_{e} P_{r}\left(-2 T_{2}\right)+c^{2}\left(T_{1}\right)-2 c\left(2 T_{2}\right)
\end{array}\right\} \\
& +\left(\frac{v}{r}\right)^{2}\left\{\begin{array}{c}
c^{2}(-a)-2 c\left(-T_{1}\right)+c^{2}(-b)+2\left(2 T_{2}\right)+c^{2}(a)-c R_{e} P_{r}\left(-T_{1}\right) \\
+c^{2}(b)-2 c\left(T_{1}\right)
\end{array}\right\} \\
& +\left(\frac{v}{r}\right)\left\{-2 c(-b)-c R_{e} P_{r}(a)-2 c(b)\right\}+\{2(-b)\}=\frac{2 E_{c} P_{r} D}{c}\left\{1-\left(\frac{c v}{r}\right)+\left(\frac{c v}{r}\right)^{2}-\left(\frac{c v}{r}\right)^{3}\right\}
\end{aligned}
$$

Equation (290) on comparing the similar coefficients yields

$$
a=\frac{2 E_{c} D}{c R_{r}}, \quad b=-\frac{E_{c} P_{r} D}{c}, \quad T_{1}=\frac{2 E_{c} P_{r} D\left(R_{e} P_{r}+2\right)}{\left(R_{e} P_{r}\right)^{2}}, \quad T_{2}=\frac{-c E_{c} D}{R_{e}}
$$

On inserting equation (291) in equation (289) we get

$$
T=\frac{2 E_{c} D}{c R_{r}} \ln r-\frac{E_{c} P_{r} D}{c} \ln v+T_{0}+\frac{2 E_{c} P_{r} D\left(R_{e} P_{r}+2\right)}{\left(R_{e} P_{r}\right)^{2}}\left(\frac{v}{r}\right)-\frac{c E_{c} D}{R_{e}}\left(\frac{v}{r}\right)^{2}
$$


For $(m, n)=(2,-2)$ the function $A, \mu$ and the generalized pressure distribution $L$ are

$$
\begin{aligned}
& A(r, \psi)=D\left(\frac{v}{r}\right)^{2} \\
& \mu=\left(\frac{-D c}{2}\right)\left(\frac{v^{3}}{(r-c v)}\right) \\
& R_{e} L=\left(\frac{R_{e}}{c^{2} v^{2}}\right)-\left(\frac{D v^{2}}{r^{2}}\right)+\left(\frac{2 D v}{c r}\right)+\left(\frac{2 D}{c^{2}}\right) \ln r+p_{8}
\end{aligned}
$$

The energy equation is

$$
\begin{aligned}
(c v)^{2} T_{r r} & -2\left(1-\frac{c v}{r}\right) c v^{2} T_{v r}+v^{2}\left\{2-\frac{2 c v}{r}+\frac{c^{2} v^{2}}{r^{2}}\right\} T_{v v}+\left(\frac{c^{2} v^{2}}{r}-c v R_{e} P_{r}\right) T_{r}+\left(-\frac{2 c v^{2}}{r}+\frac{c^{2} v^{3}}{r^{2}}\right) T_{v} \\
& =\frac{2 D E_{c} P_{r}}{c}\left[\left(\frac{v}{r}\right)-c\left(\frac{v}{r}\right)^{2}+c^{2}\left(\frac{v}{r}\right)^{3}-c^{3}\left(\frac{v}{r}\right)^{4}\right]
\end{aligned}
$$

which on substituting

$$
T=a \ln r+T_{0}+T_{1}\left(\frac{v}{r}\right)+T_{2}\left(\frac{v}{r}\right)^{2}+T_{3}\left(\frac{v}{r}\right)^{3}
$$

yields

$$
a=-\frac{2 E_{c} D}{c^{2} R_{e}}, \quad T_{1}=\frac{-E_{c} D}{c R_{e}}\left(1+\frac{4}{R_{e} P_{r}}-\frac{8}{\left(R_{e} P_{r}\right)^{2}}\right), \quad T_{2}=\frac{E_{c} D}{R_{e}}\left(1+\frac{4}{R_{e} P_{r}}\right), \quad \text { and } \quad T_{3}=\frac{-2 E_{c} D}{3 R_{e}}
$$

Substituting equation (298) in equation (297) we get

$$
T=\left(-\frac{2 E_{c} D}{c^{2} R_{e}}\right) \ln r+T_{0}+\left(\frac{-E_{c} D}{c R_{e}}\right)\left(1+\frac{4}{R_{e} P_{r}}-\frac{8}{\left(R_{e} P_{r}\right)^{2}}\right)\left(\frac{v}{r}\right)+\left(\frac{E_{c} D}{R_{e}}\right)\left(1+\frac{4}{R_{e} P_{r}}\right)\left(\frac{v}{r}\right)^{2}+\left(\frac{-2 E_{c} D}{3 R_{e}}\right)\left(\frac{v}{r}\right)^{3}
$$

\section{Results and discussion}

For the flows under consideration the streamlines are given by $\frac{\theta-f(r)}{g(r)}=$ Const. for case $\mathrm{I}, g(r)=1$ the streamlines are $\theta-f(r)=$ Const. When $f(r)$ is arbitrary we can construct an infinite set of streamlines and also an infinite set of velocity components. This indicates an infinite set of solutions to the flow equations. When $f(r)$ is not arbitrary there are two values of $f(r)$, and therefore, two solutions to flow equations. The streamlines for case I are plotted in Fig. (1-16). The Fig. (1-16) shows the effect of different chosen forms of $f(r)$.

For case II when $f(r)=0, g(r)=\frac{-1}{c_{0} r^{2}+c_{1}}$ the streamlines are presented through Fig.(17-25) and the influence of various parameters are also indicated. When $f(r)$ is non-zero and $g(r)=\frac{c}{r}$, we can construct infinite set of velocity components and streamlines since $f(r)$ is arbitrary. This indicates an infinite set of solutions to flow equations. The Fig. (26-50) clearly indicate the effect on streamlines for different forms of $f(r)$.

When $v(\psi)=e^{\psi}$ there are two values of $g(r)$. When $g(r)=\frac{c}{r}$ the function $f(r)=\ln r+m_{1}$. The streamlines for $g(r)=\frac{c}{r}$ and $f(r)=\ln r+m_{1}$ are presented through Fig. (51-56).

\section{Conclusion}

The aim of this paper is to indicate a class of new exact solutions of the equations governing the steady plane flows of incompressible fluid of variable viscosity in the absence of external force. To achieve our aim, we first transformed the flow equation into Martin system $(\phi, \psi)$, and then setting $\phi$ defined in equation (34). The exact solutions are determined 
when $f(r)$ is arbitrary and $f(r)$ is not arbitrary. When $f(r)$ is arbitrary an infinite set of velocity component implying an infinite solution to flow equations. When $f(r)$ is not arbitrary, there are solutions of the flow equation. We see that in case II $f(r)=0$, and there is solution and when $f(r) \neq 0$, we find that $f(r)$ is arbitrary, and therefore, we can construct an infinite set of solutions. The influences of various chosen forms of $f(r)$ on the streamlines are also presented.

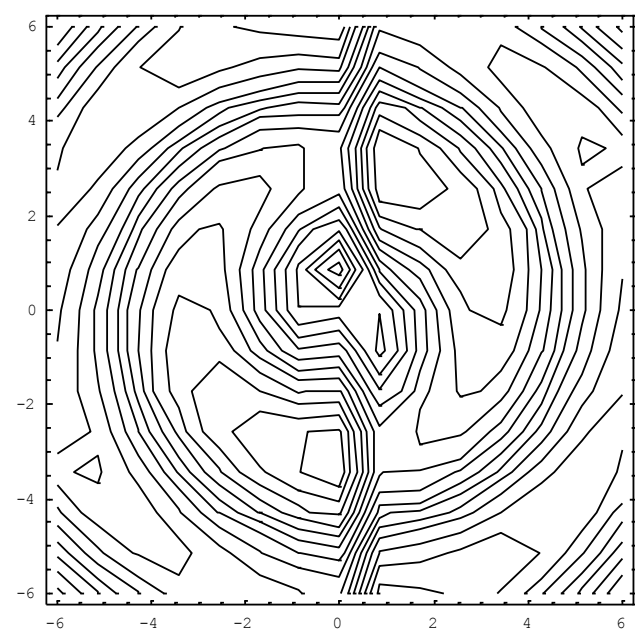

Fig. 1: Streamlines Pattern for $\operatorname{Tan}^{-1}\left(\frac{y}{x}\right)-2 \operatorname{Cos} \sqrt{x^{2}+y^{2}}-1$

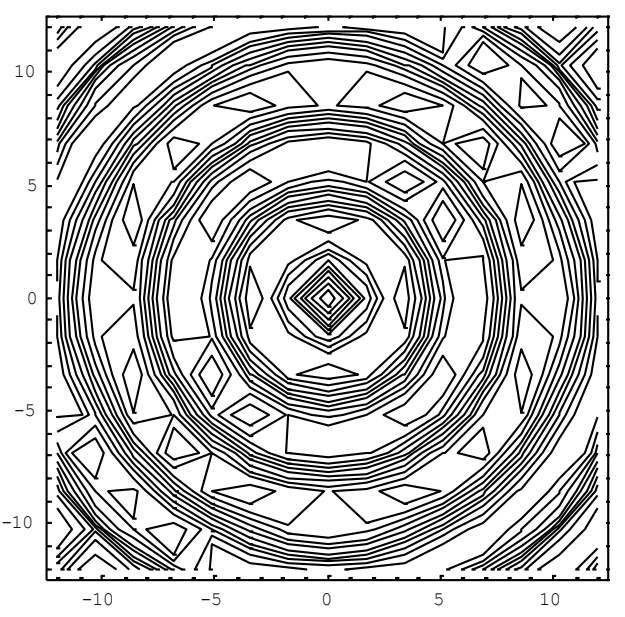

Fig. 3: Streamlines Pattern for $\operatorname{Tan}^{-1}\left(\frac{y}{x}\right)-280 \operatorname{Cos} \sqrt{x^{2}+y^{2}}-1$

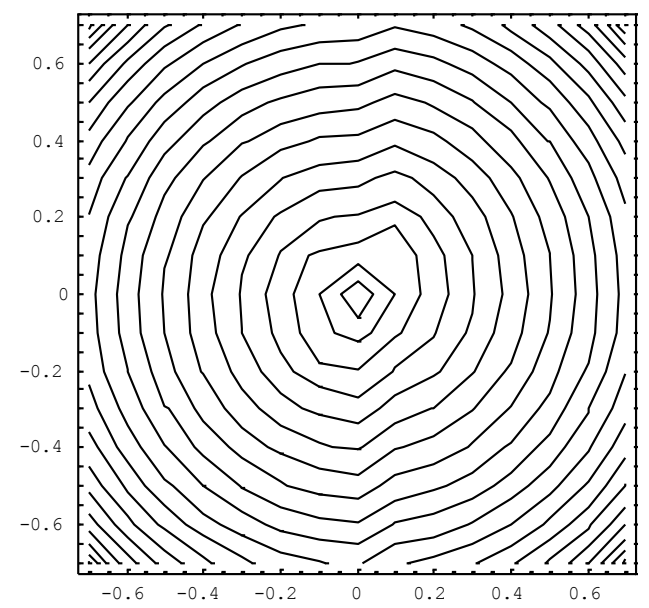

Fig. 5: Streamlines Pattern for $\operatorname{Tan}^{-1}\left(\frac{y}{x}\right)+50 \operatorname{Cos}^{-1} \sqrt{x^{2}+y^{2}}-200$

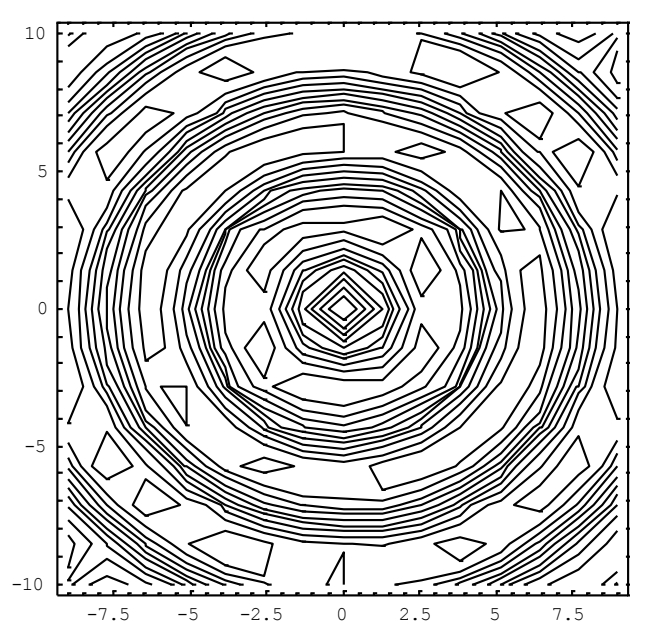

Fig. 2: Streamlines Pattern for $\operatorname{Tan}^{-1}\left(\frac{y}{x}\right)-28 \operatorname{Cos} \sqrt{x^{2}+y^{2}}-1$

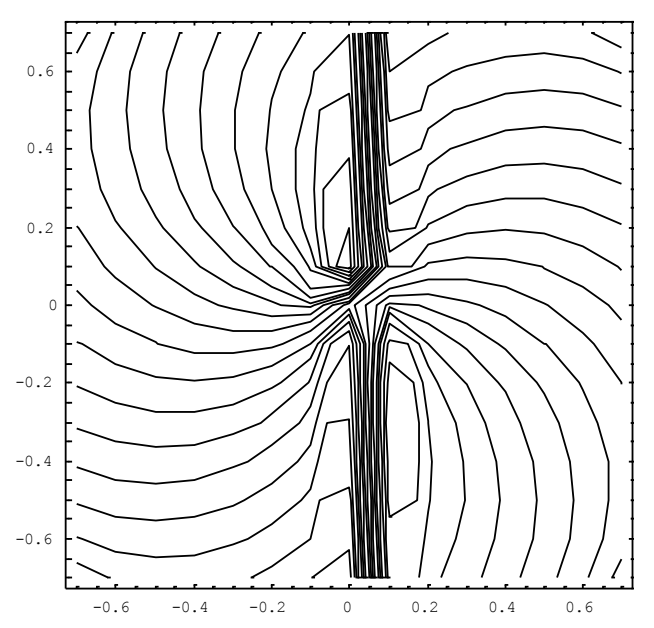

Fig. 4: Streamlines Pattern for $\operatorname{Tan}^{-1}\left(\frac{y}{x}\right)-\operatorname{Cos}^{-1} \sqrt{x^{2}+y^{2}}+1$

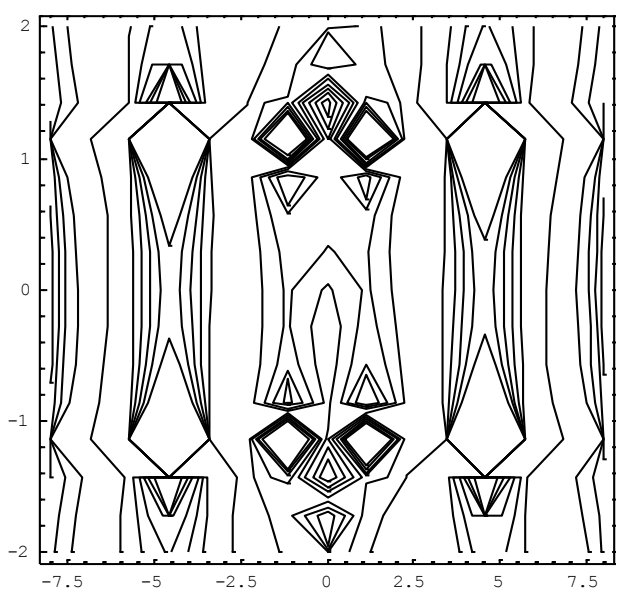

Fig. 6: Streamlines Pattern for $\operatorname{Tan}^{-1}\left(\frac{y}{x}\right)-\operatorname{Tan}\left(\sqrt{x^{2}+y^{2}}\right)-1$ 


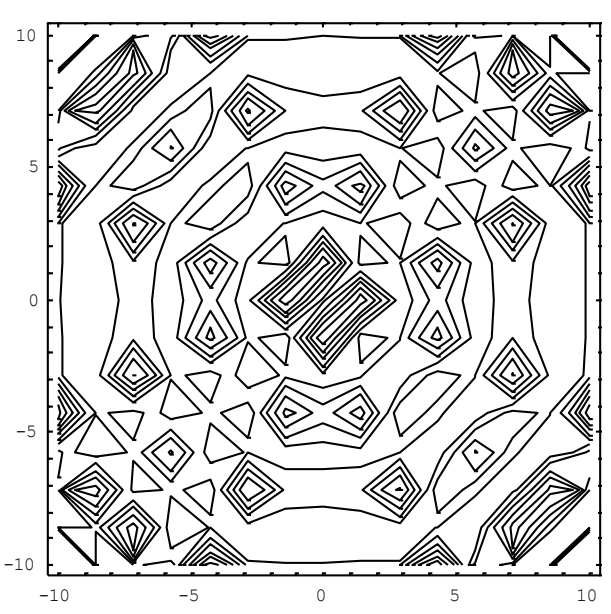

Fig. 7: Streamlines Pattern for $\operatorname{Tan}^{-1}\left(\frac{y}{x}\right)-15 \operatorname{Tan}\left(\sqrt{x^{2}+y^{2}}\right)-1$

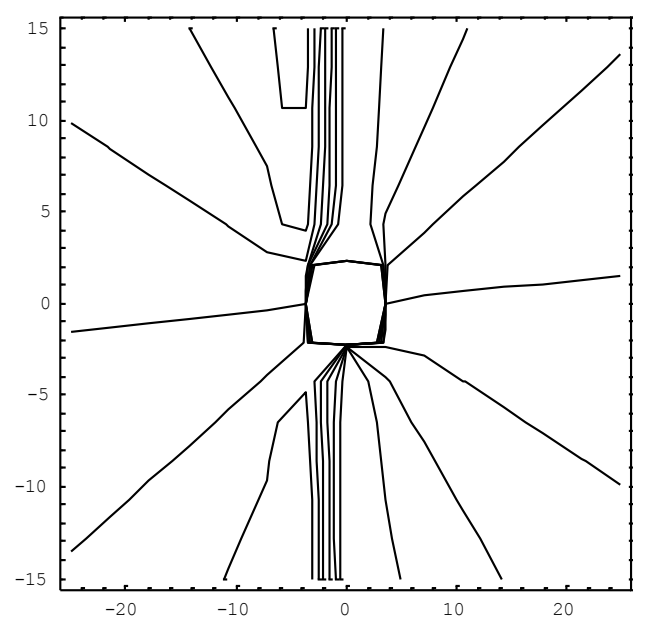

Fig. 9: Streamlines Pattern for $\operatorname{Tan}^{-1}\left(\frac{y}{x}\right)-500 \operatorname{Tanh} \sqrt{x^{2}+y^{2}}-1$

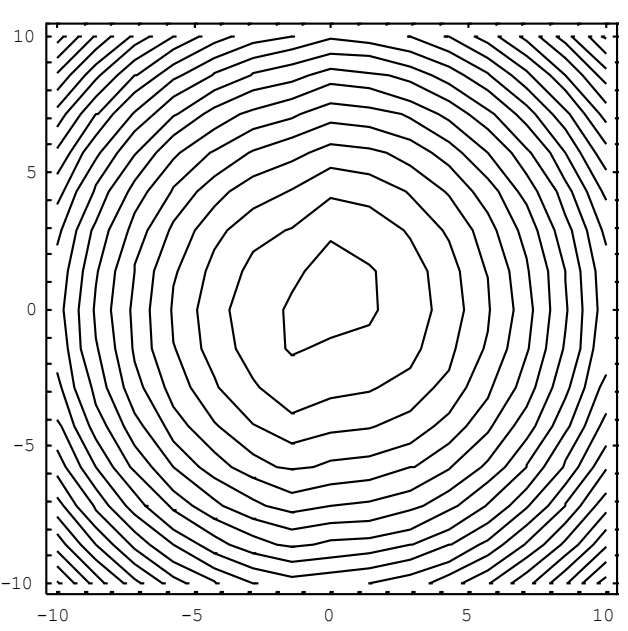

Fig. 11: Streamlines Pattern for $\operatorname{Tan}^{-1}\left(\frac{y}{x}\right)-\frac{1}{2}\left(x^{2}+y^{2}\right)-1$

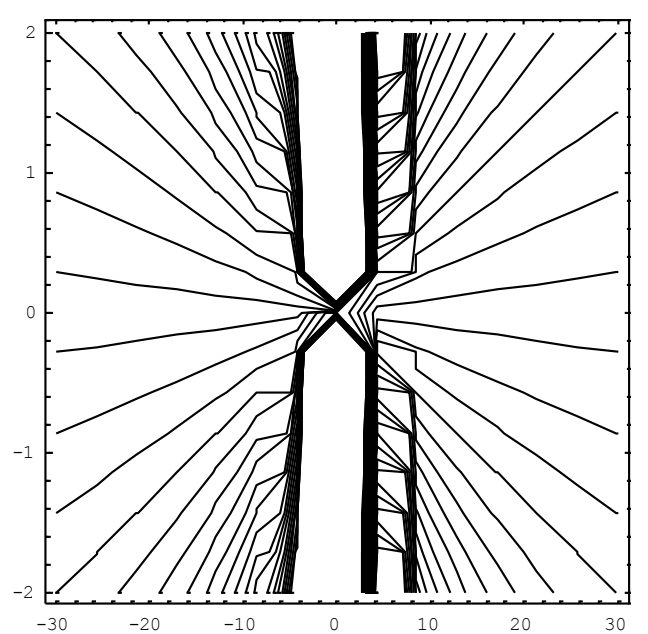

Fig. 8: Streamlines Pattern for $\operatorname{Tan}^{-1}\left(\frac{y}{x}\right)-(0.001) \operatorname{Tan}^{-1}\left(\sqrt{x^{2}+y^{2}}\right)-5$

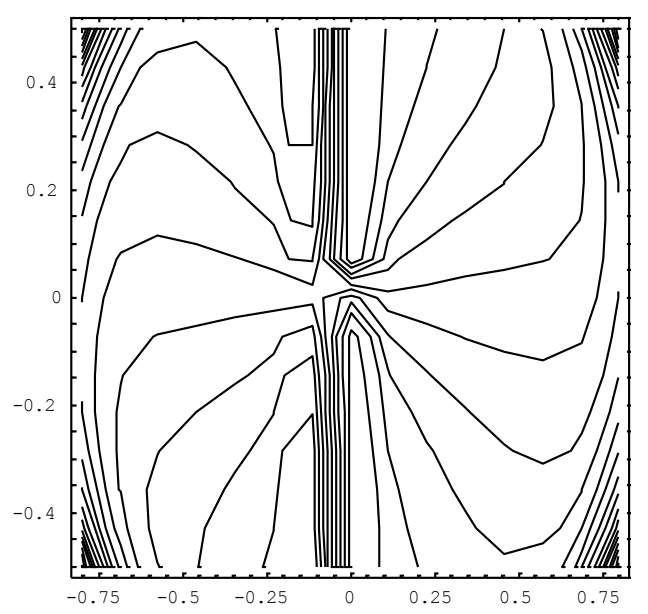

Fig. 10: Streamlines Pattern for $\operatorname{Tan}^{-1}\left(\frac{y}{x}\right)-8 \operatorname{Tanh}^{-1} \sqrt{x^{2}+y^{2}}-5$

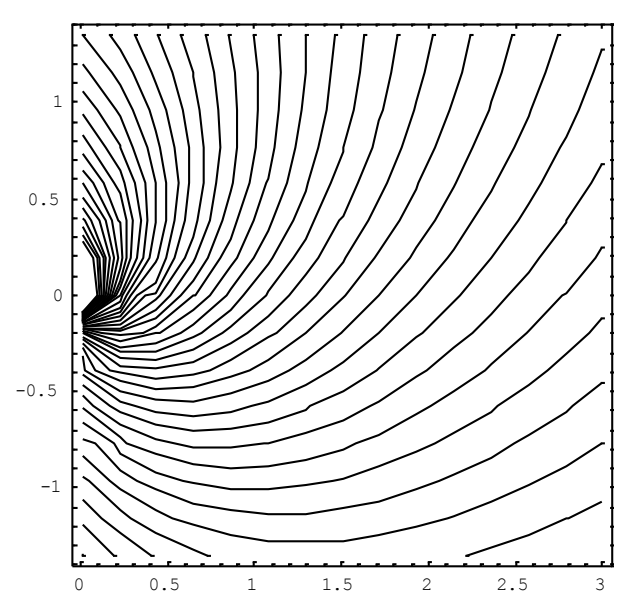

Fig. 12: Streamlines Pattern for $\operatorname{Tan}^{-1}\left(\frac{y}{x}\right)-\ln \sqrt{x^{2}+y^{2}}-\frac{1}{120}$ 


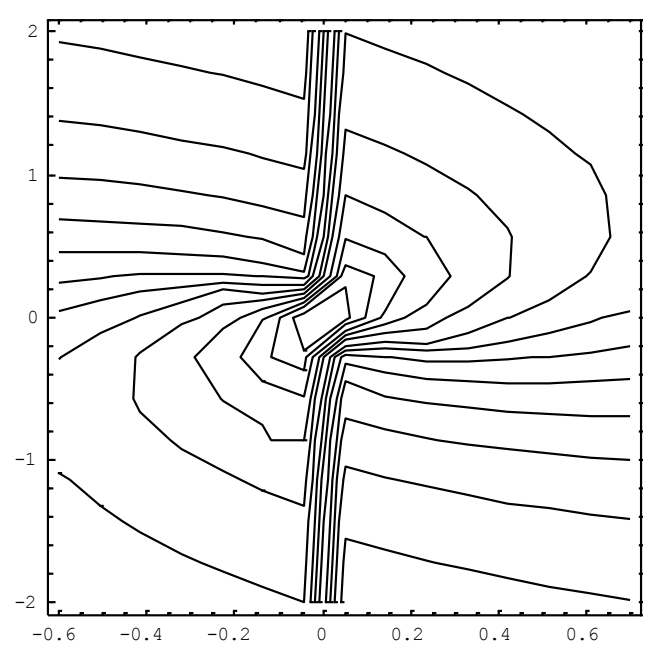

Fig. 13: Streamlines Pattern for $\operatorname{Tan}^{-1}\left(\frac{y}{x}\right)-\ln \sqrt{x^{2}+y^{2}}-\frac{1}{50}$

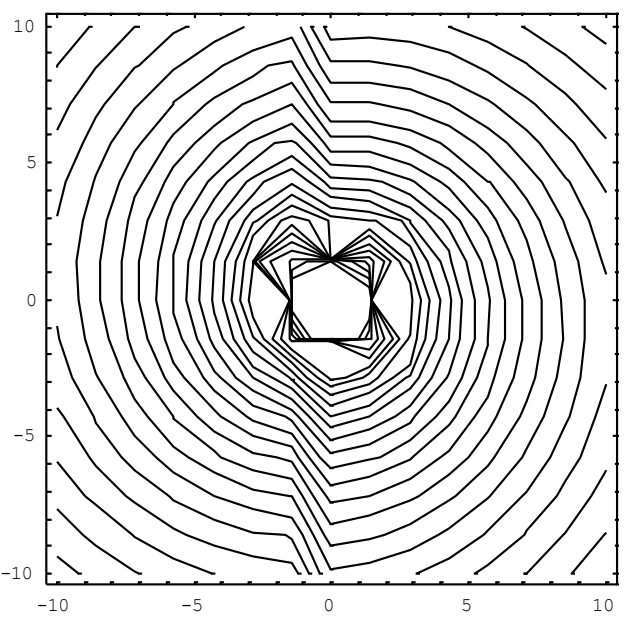

Fig. 15: Streamlines Pattern for $\operatorname{Tan}^{-1}\left(\frac{y}{x}\right)-10 \ln \sqrt{x^{2}+y^{2}}-100$

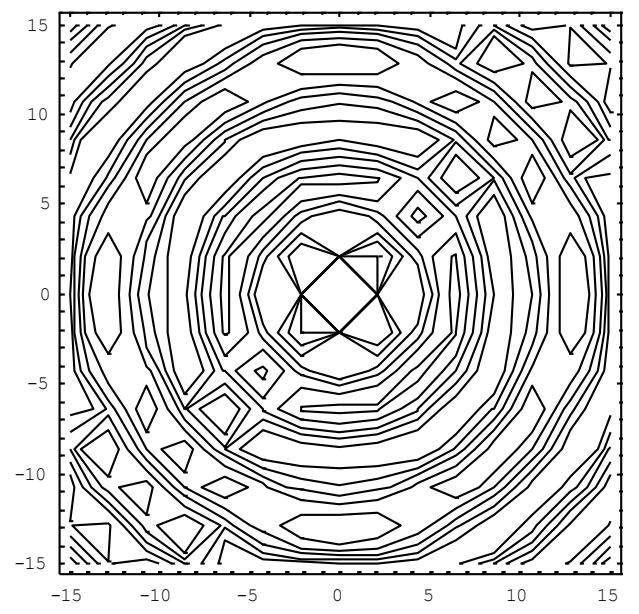

Fig. 26: Streamlines Pattern for

$\operatorname{Tan}^{-1}\left(\frac{y}{x}\right)+300 \operatorname{Cos} \sqrt{x^{2}+y^{2}}+\frac{500}{\sqrt{x^{2}+y^{2}}}$

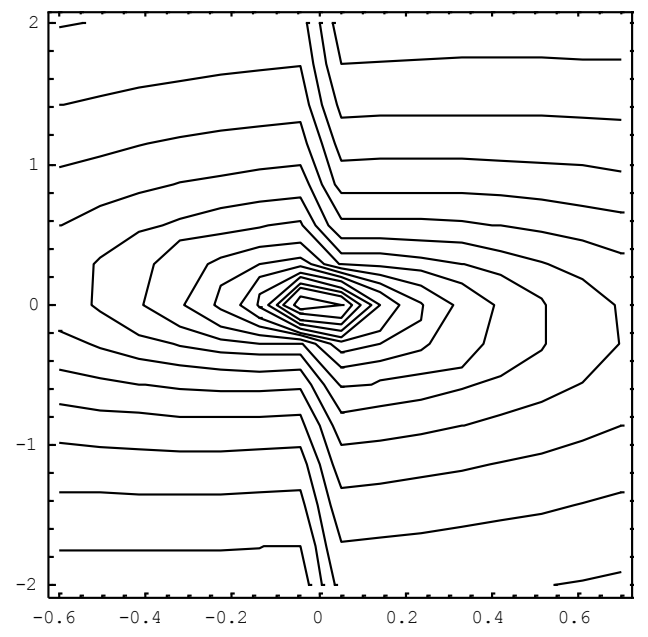

Fig. 14: Streamlines Pattern for $\operatorname{Tan}^{-1}\left(\frac{y}{x}\right)+4 \ln \sqrt{x^{2}+y^{2}}-1$

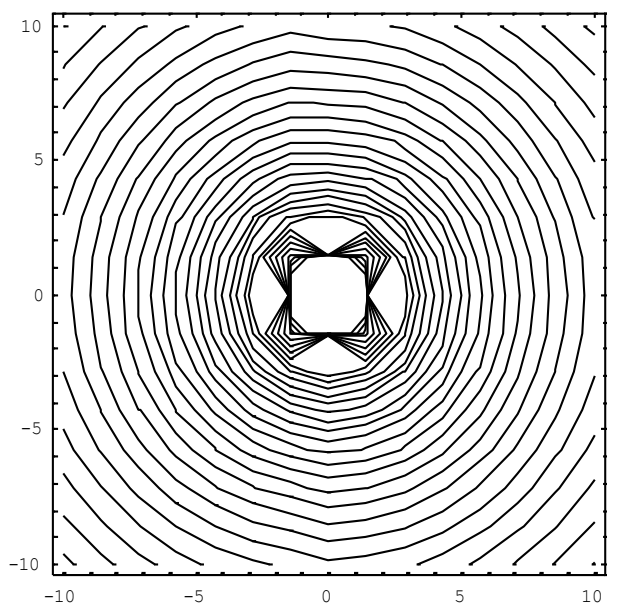

Fig. 16: Streamlines Pattern for $\operatorname{Tan}^{-1}\left(\frac{y}{x}\right)+100 \ln \sqrt{x^{2}+y^{2}}-1$

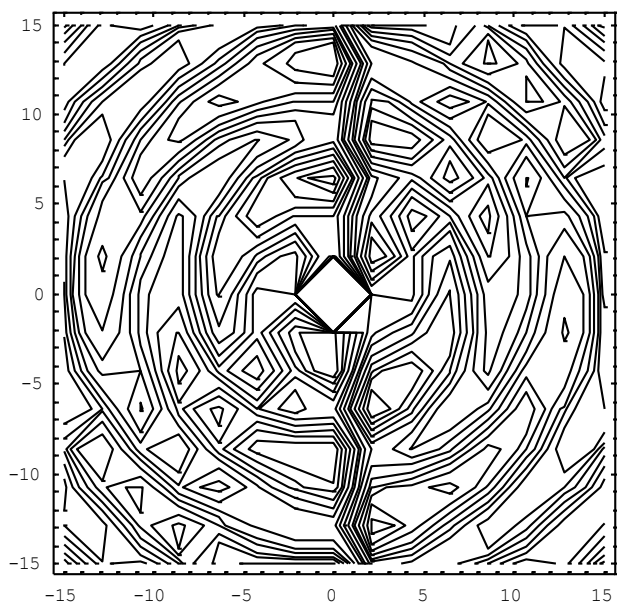

Fig. 27: Streamlines Pattern for $\operatorname{Tan}^{-1}\left(\frac{y}{x}\right)+\operatorname{Cos} \sqrt{x^{2}+y^{2}}-\frac{1}{\sqrt{x^{2}+y^{2}}}$ 


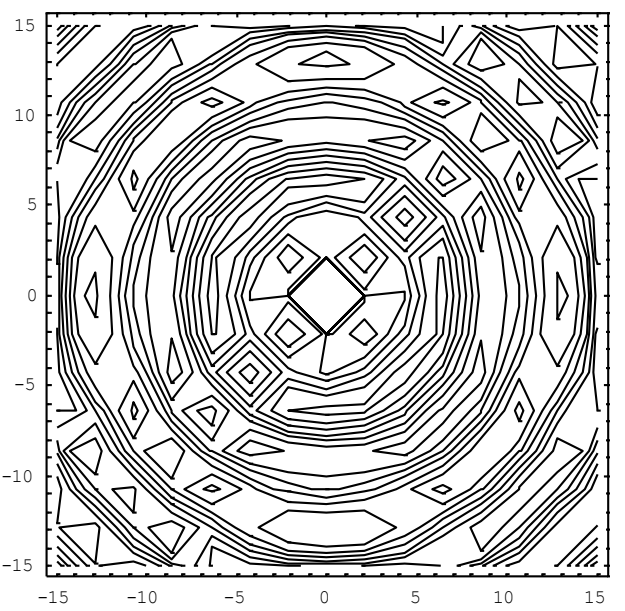

Fig. 28: Streamlines Pattern for $\operatorname{Tan}^{-1}\left(\frac{y}{x}\right)+100 \operatorname{Cos} \sqrt{x^{2}+y^{2}}-\frac{100}{\sqrt{x^{2}+y^{2}}}$

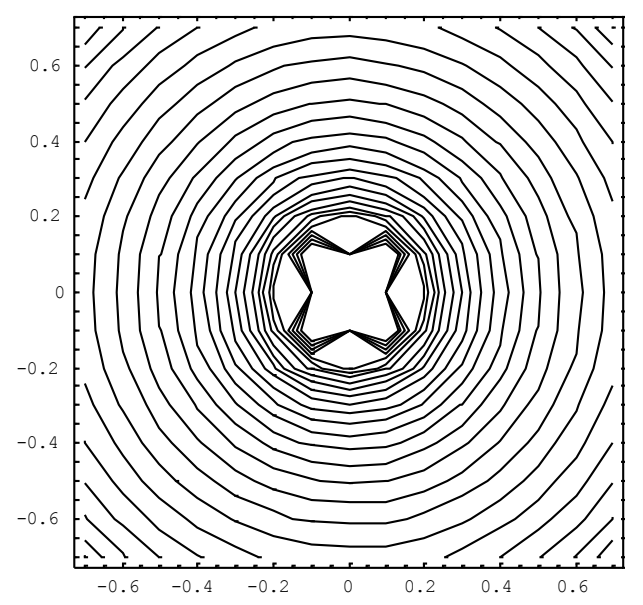

Fig. 30: Streamlines Pattern for $\operatorname{Tan}^{-1}\left(\frac{y}{x}\right)-200 \operatorname{Cos}^{-1} \sqrt{x^{2}+y^{2}}-\frac{100}{\sqrt{x^{2}+y^{2}}}$

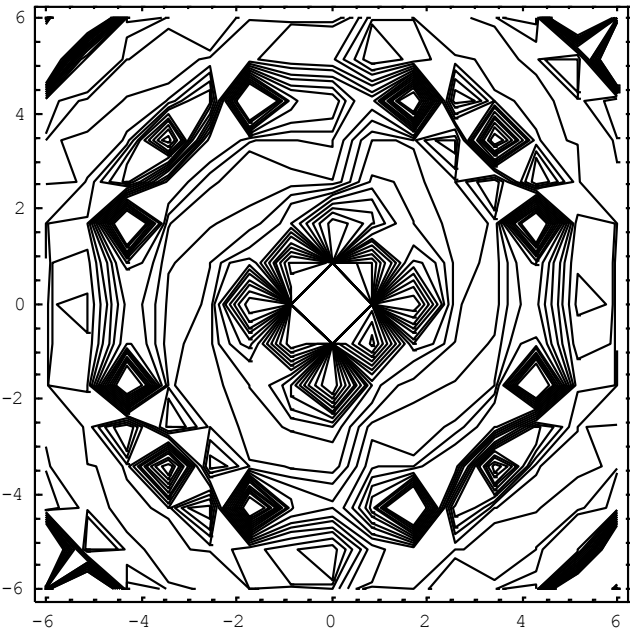

Fig. 32: Streamlines Pattern for $\operatorname{Tan}^{-1}\left(\frac{y}{x}\right)-\operatorname{Tan} \sqrt{x^{2}+y^{2}}-\frac{1}{\sqrt{x^{2}+y^{2}}}$

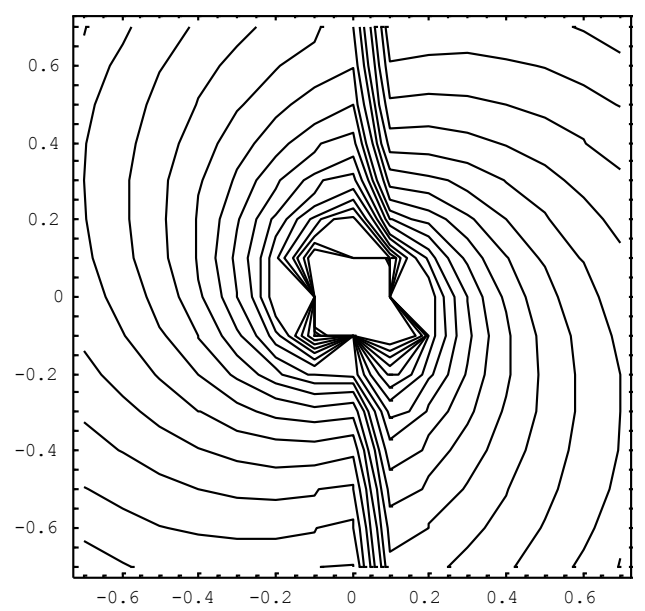

Fig. 29: Streamlines Pattern for

$\operatorname{Tan}^{-1}\left(\frac{y}{x}\right)-\operatorname{Cos}^{-1} \sqrt{x^{2}+y^{2}}-\frac{1}{\sqrt{x^{2}+y^{2}}}$

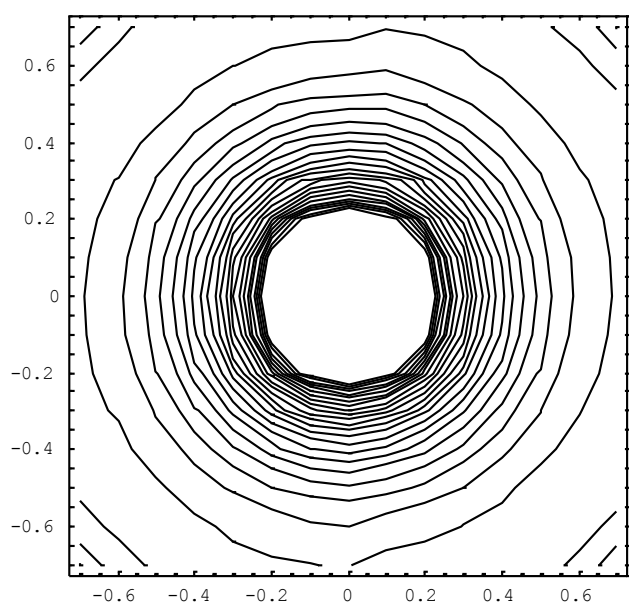

Fig. 31: Streamlines Pattern for

$\operatorname{Tan}^{-1}\left(\frac{y}{x}\right)-100 \operatorname{Cos}^{-1} \sqrt{x^{2}+y^{2}}+\frac{100}{\sqrt{x^{2}+y^{2}}}$

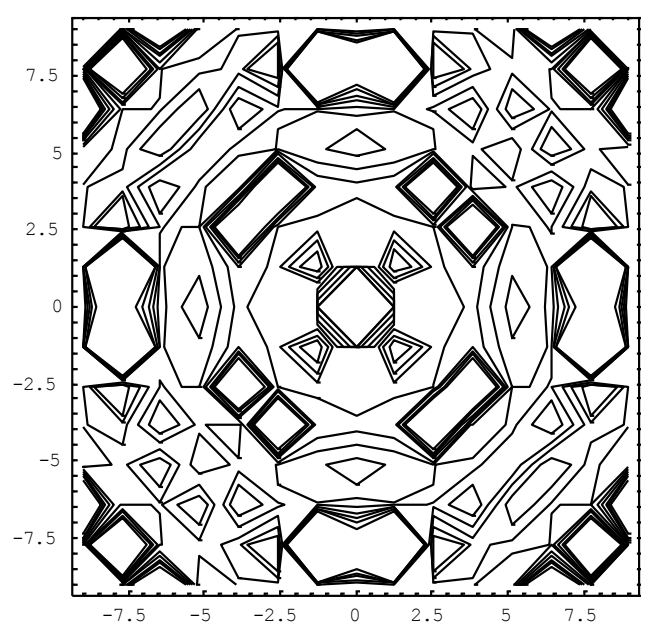

Fig. 33: Streamlines Pattern for

$\operatorname{Tan}^{-1}\left(\frac{y}{x}\right)-200 \operatorname{Tan} \sqrt{x^{2}+y^{2}}-\frac{100}{\sqrt{x^{2}+y^{2}}}$ 


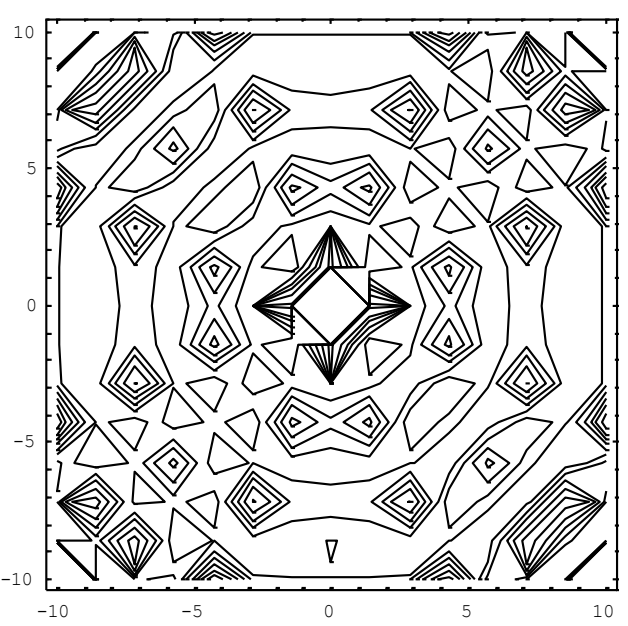

Fig. 34: Streamlines Pattern for $\operatorname{Tan}^{-1}\left(\frac{y}{x}\right)+100 \operatorname{Tan} \sqrt{x^{2}+y^{2}}-\frac{100}{\sqrt{x^{2}+y^{2}}}$

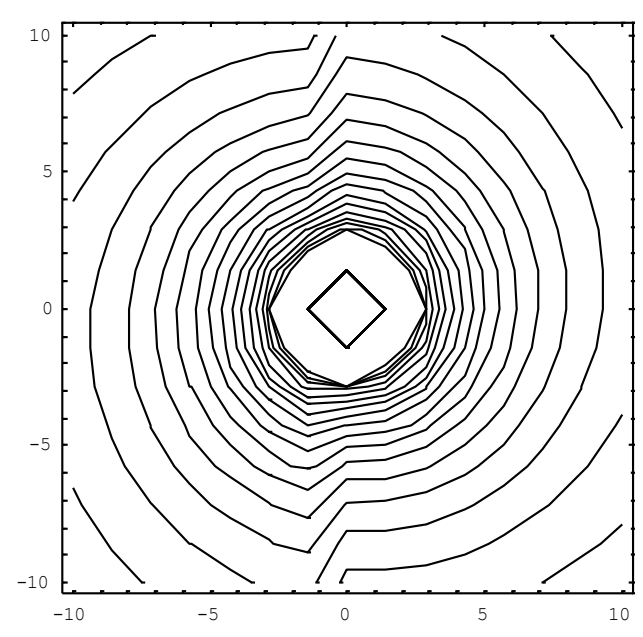

Fig. 36: Streamlines Pattern for

$\operatorname{Tan}^{-1}\left(\frac{y}{x}\right)-200 \operatorname{Tan}^{-1} \sqrt{x^{2}+y^{2}}-\frac{100}{\sqrt{x^{2}+y^{2}}}$

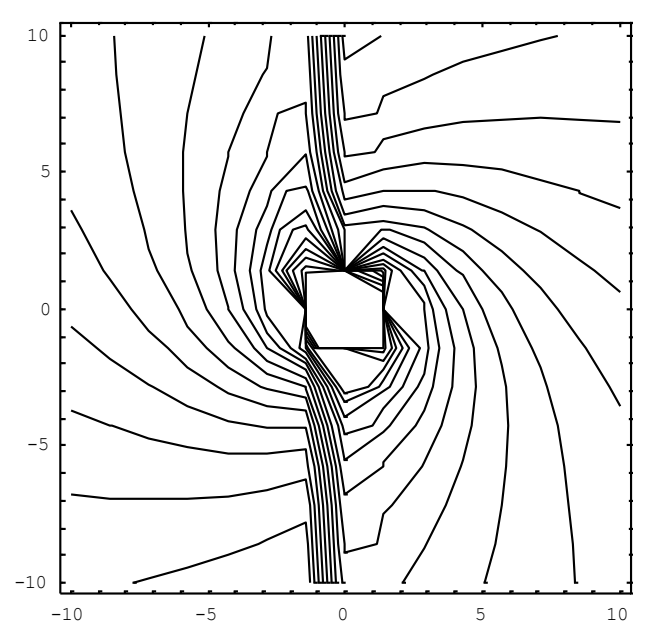

Fig. 38: Streamlines Pattern for

$$
\operatorname{Tan}^{-1}\left(\frac{y}{x}\right)-\operatorname{Tanh} \sqrt{x^{2}+y^{2}}-\frac{10}{\sqrt{x^{2}+y^{2}}}
$$

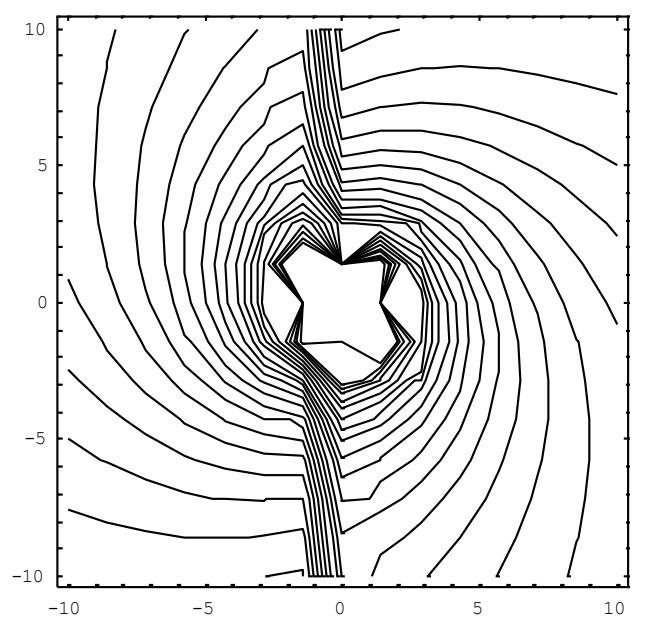

Fig. 35: Streamlines Pattern for

$\operatorname{Tan}^{-1}\left(\frac{y}{x}\right)-\operatorname{Tan}^{-1} \sqrt{x^{2}+y^{2}}-\frac{20}{\sqrt{x^{2}+y^{2}}}$

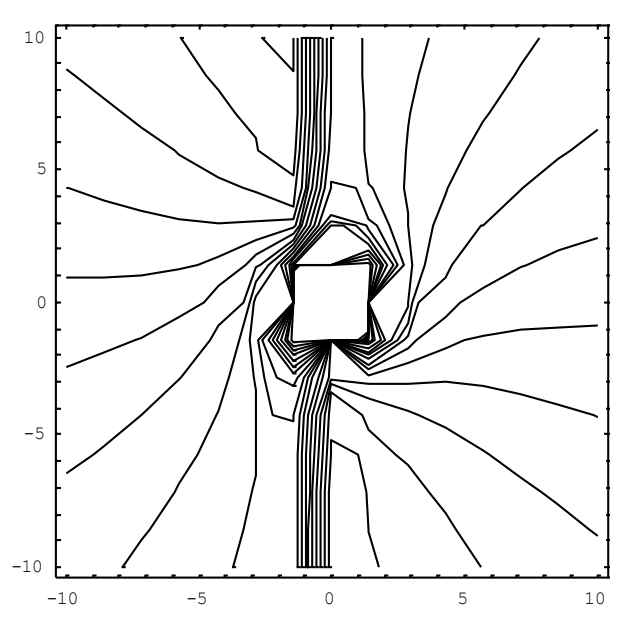

Fig. 37: Streamlines Pattern for

$\operatorname{Tan}^{-1}\left(\frac{y}{x}\right)-100 \operatorname{Tan}^{-1} \sqrt{x^{2}+y^{2}}+\frac{100}{\sqrt{x^{2}+y^{2}}}$

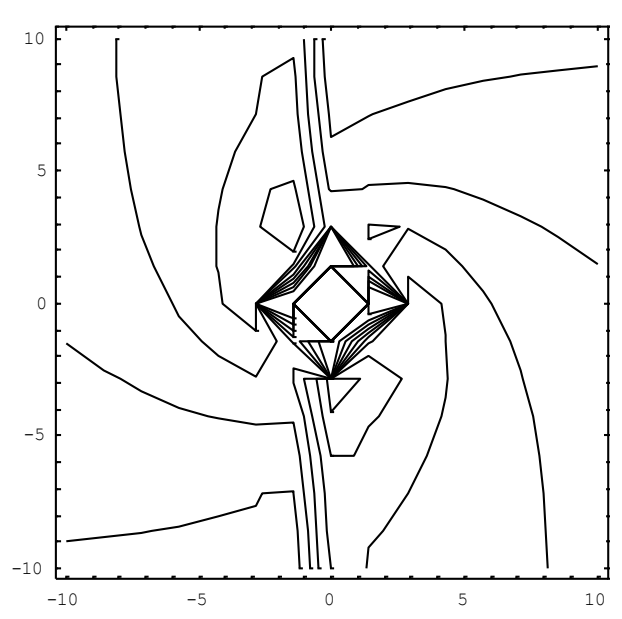

Fig. 39: Streamlines Pattern for

$\operatorname{Tan}^{-1}\left(\frac{y}{x}\right)-100 \operatorname{Tanh} \sqrt{x^{2}+y^{2}}-\frac{10}{\sqrt{x^{2}+y^{2}}}$ 


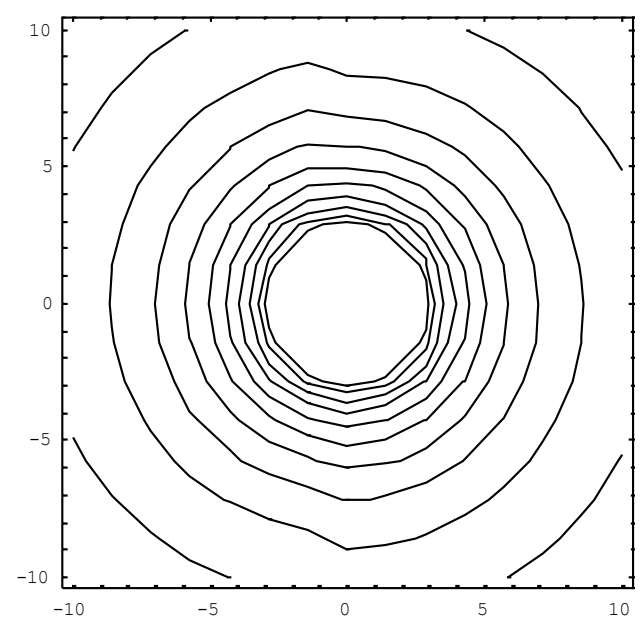

Fig. 40: Streamlines Pattern for $\operatorname{Tan}^{-1}\left(\frac{y}{x}\right)-2 \operatorname{Tanh} \sqrt{x^{2}+y^{2}}-\frac{400}{\sqrt{x^{2}+y^{2}}}$

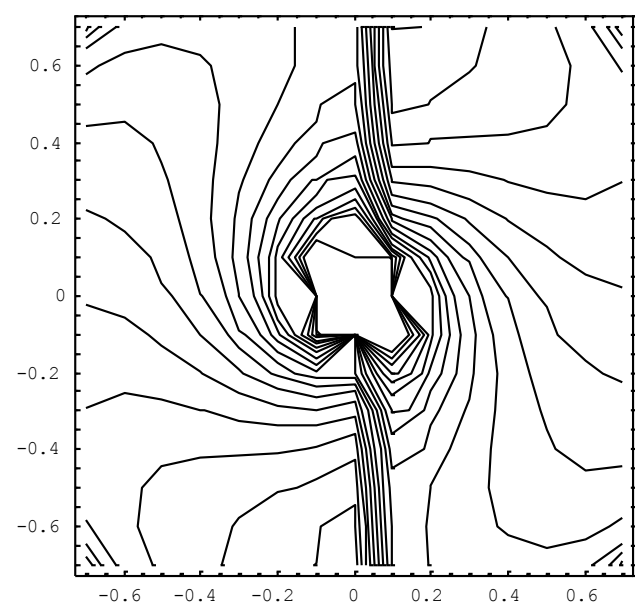

Fig. 42: Streamlines Pattern for

$\operatorname{Tan}^{-1}\left(\frac{y}{x}\right)-\operatorname{Tanh}^{-1} \sqrt{x^{2}+y^{2}}-\frac{1}{\sqrt{x^{2}+y^{2}}}$

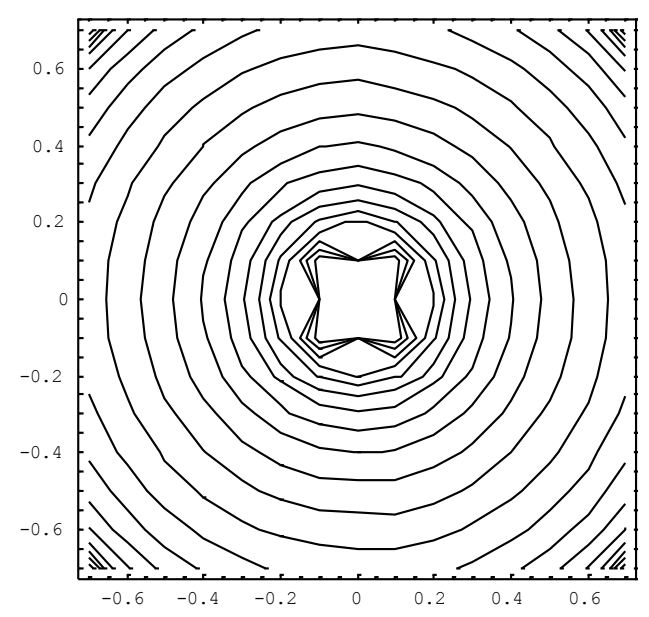

Fig. 44: Streamlines Pattern for

$\operatorname{Tan}^{-1}\left(\frac{y}{x}\right)+100 \operatorname{Tanh}^{-1} \sqrt{x^{2}+y^{2}}-\frac{40}{\sqrt{x^{2}+y^{2}}}$

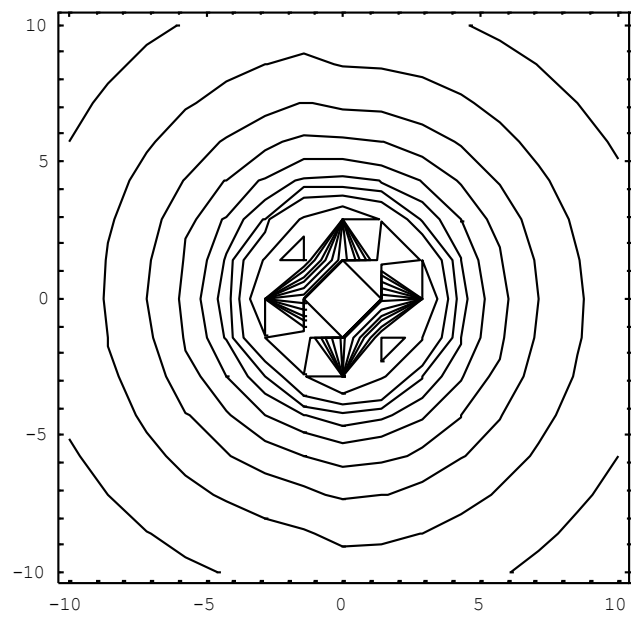

Fig. 41: Streamlines Pattern for

$\operatorname{Tan}^{-1}\left(\frac{y}{x}\right)-2000 \operatorname{Tanh} \sqrt{x^{2}+y^{2}}-\frac{400}{\sqrt{x^{2}+y^{2}}}$

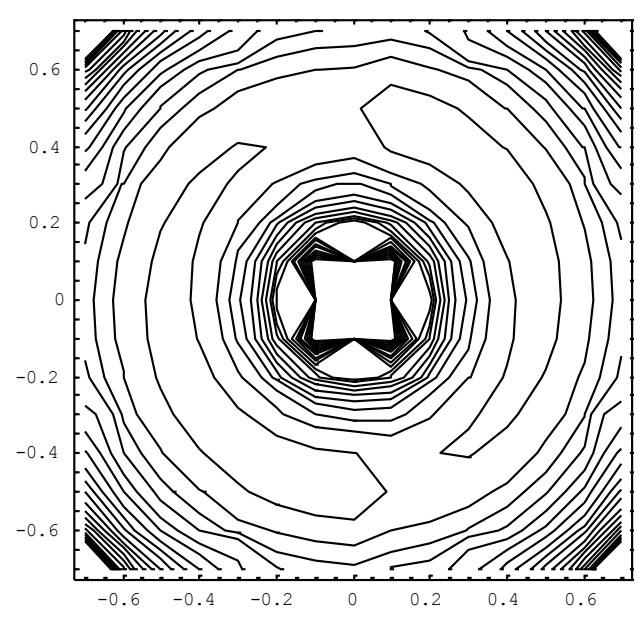

Fig. 43: Streamlines Pattern for

$\operatorname{Tan}^{-1}\left(\frac{y}{x}\right)-100 \operatorname{Tanh}^{-1} \sqrt{x^{2}+y^{2}}-\frac{30}{\sqrt{x^{2}+y^{2}}}$

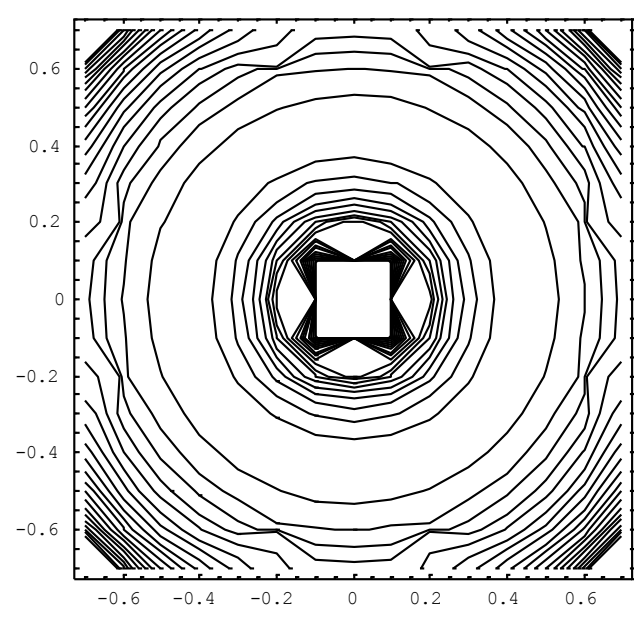

Fig. 45: Streamlines Pattern for

$\operatorname{Tan}^{-1}\left(\frac{y}{x}\right)+2000 \operatorname{Tanh}^{-1} \sqrt{x^{2}+y^{2}}-\frac{500}{\sqrt{x^{2}+y^{2}}}$ 


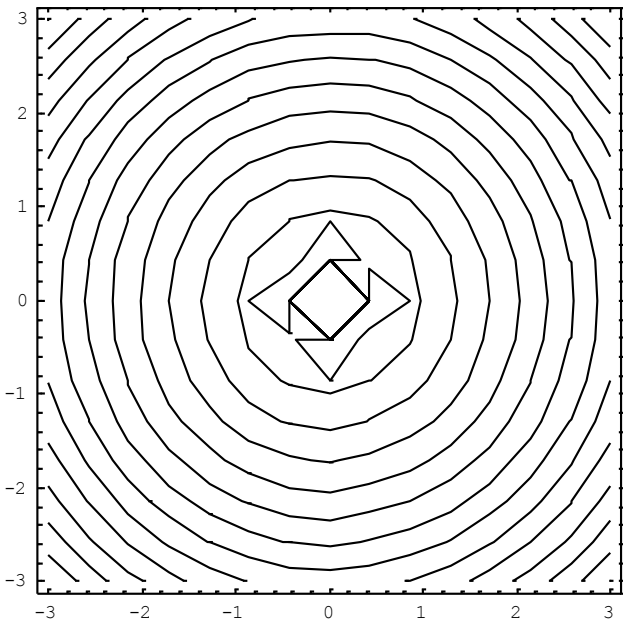

Fig. 46: Streamlines Pattern for

$\operatorname{Tan}^{-1}\left(\frac{y}{x}\right)+10\left(x^{2}+y^{2}\right)-50 \sqrt{\left(x^{2}+y^{2}\right)}-10-\frac{1}{\sqrt{x^{2}+y^{2}}}$

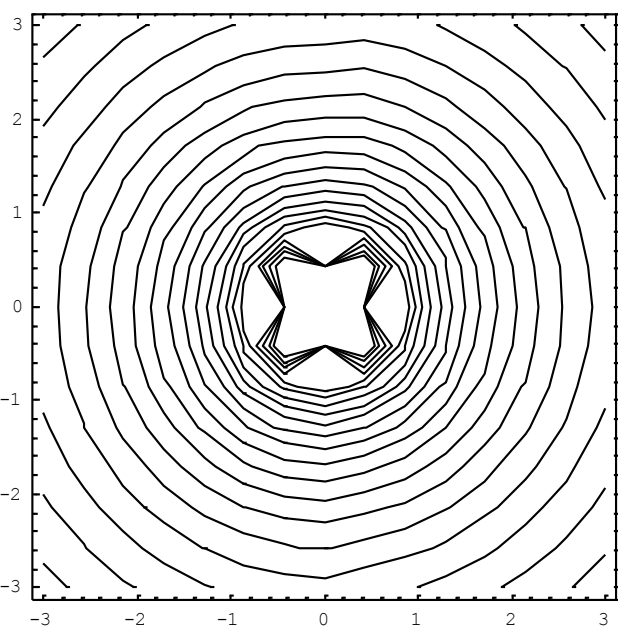

Fig. 48: Streamlines Pattern for

$\operatorname{Tan}^{-1}\left(\frac{y}{x}\right)-10\left[\ln \sqrt{\left(x^{2}+y^{2}\right)}\right]^{2}-50 \ln \sqrt{\left(x^{2}+y^{2}\right)}-10+\frac{100}{\sqrt{x^{2}+y^{2}}}$

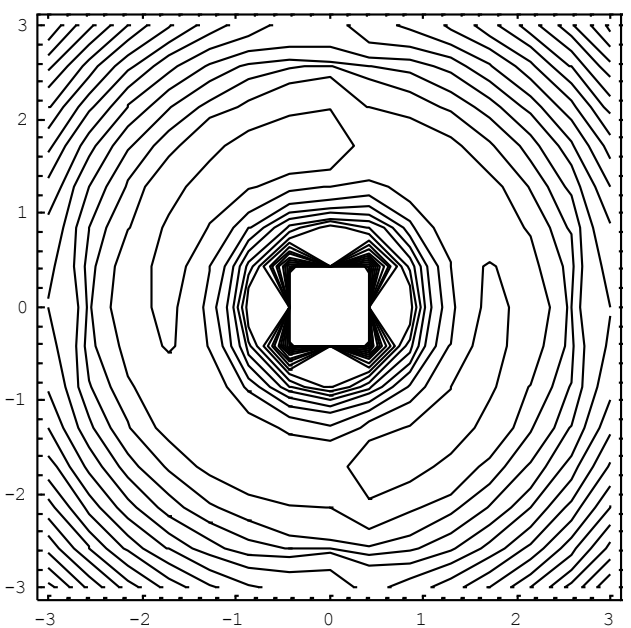

Fig. 50: Streamlines Pattern for

$\operatorname{Tan}^{-1}\left(\frac{y}{x}\right)-60\left[\ln \sqrt{\left(x^{2}+y^{2}\right)}\right]^{2}+50 \ln \sqrt{\left(x^{2}+y^{2}\right)}-1+\frac{50}{\sqrt{x^{2}+y^{2}}}$

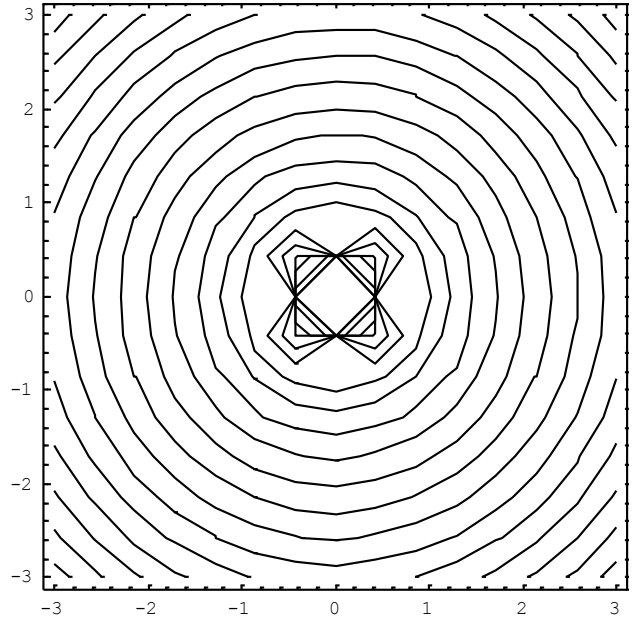

Fig. 47: Streamlines Pattern for

$\operatorname{Tan}^{-1}\left(\frac{y}{x}\right)-10\left(x^{2}+y^{2}\right)-50 \sqrt{\left(x^{2}+y^{2}\right)}-10+\frac{100}{\sqrt{x^{2}+y^{2}}}$

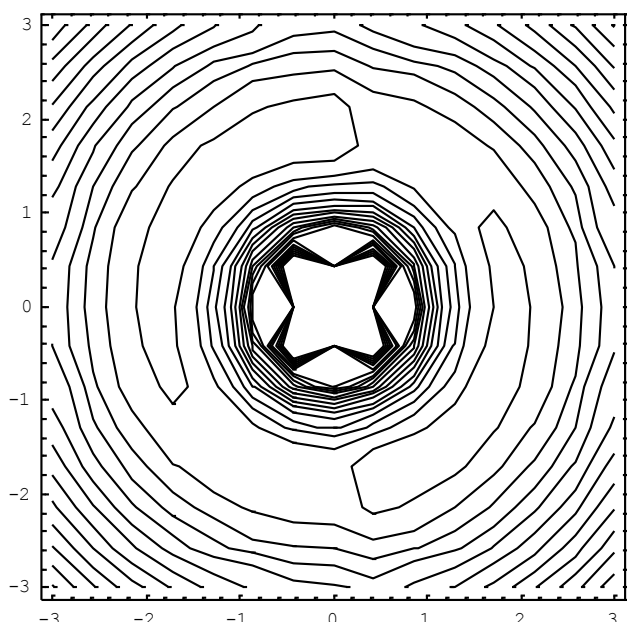

Fig. 49: Streamlines Pattern for

$\operatorname{Tan}^{-1}\left(\frac{y}{x}\right)+60\left[\ln \sqrt{\left(x^{2}+y^{2}\right)}\right]^{2}-50 \ln \sqrt{\left(x^{2}+y^{2}\right)}-1+\frac{50}{\sqrt{x^{2}+y^{2}}}$

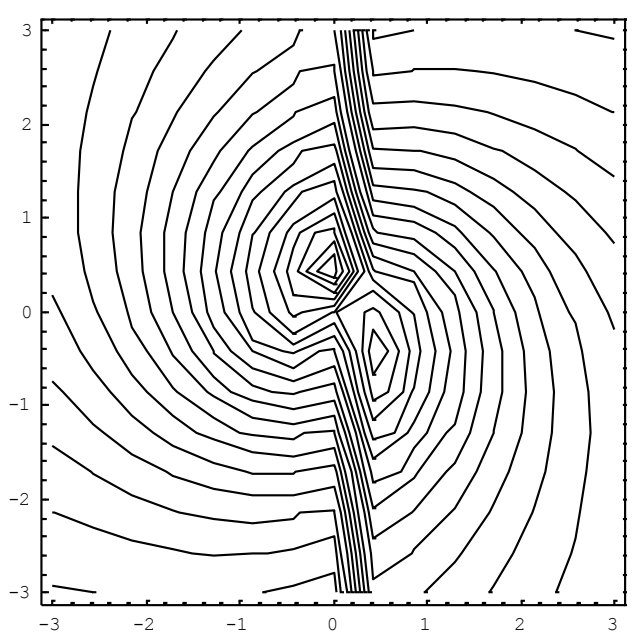

Fig. 17: Streamlines Pattern for $\operatorname{Tan}^{-1}\left(\frac{y}{x}\right)-\frac{17.8}{\left(x^{2}+y^{2}\right)+3}$ 


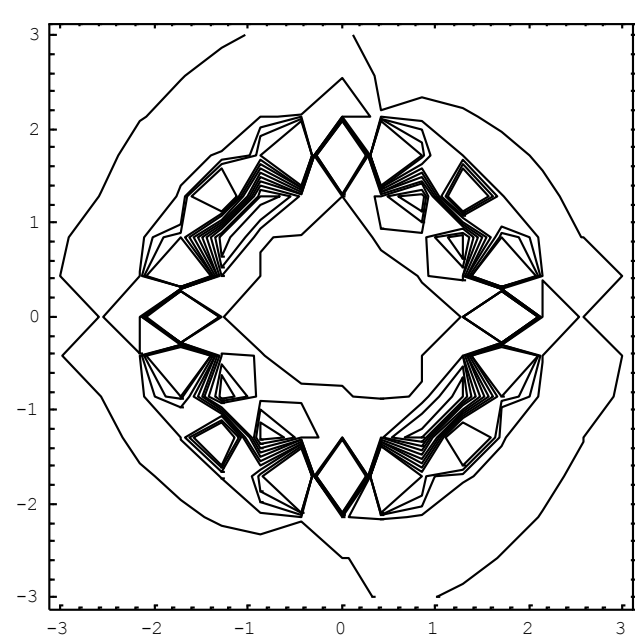

Fig. 18: Streamlines Pattern for $\operatorname{Tan}^{-1}\left(\frac{y}{x}\right)-\frac{17.8}{\left(x^{2}+y^{2}\right)-3}$

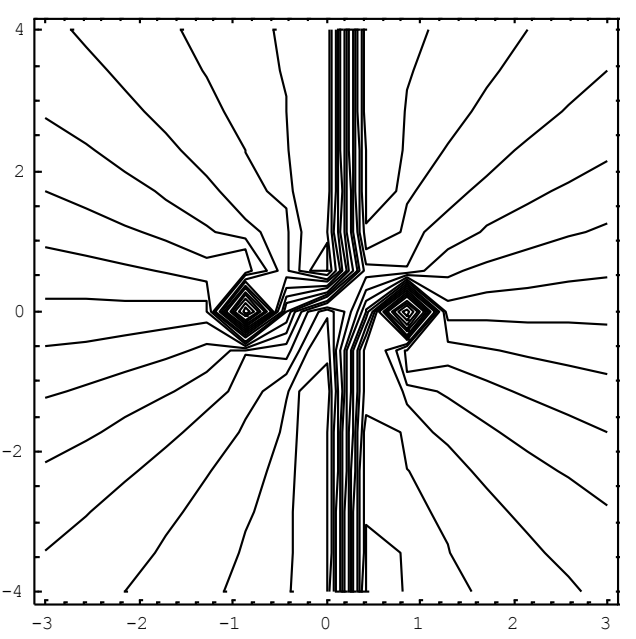

Fig. 20: Streamlines Pattern for $\operatorname{Tan}^{-1}\left(\frac{y}{x}\right)-\frac{17.8}{-400\left(x^{2}+y^{2}\right)+300}$

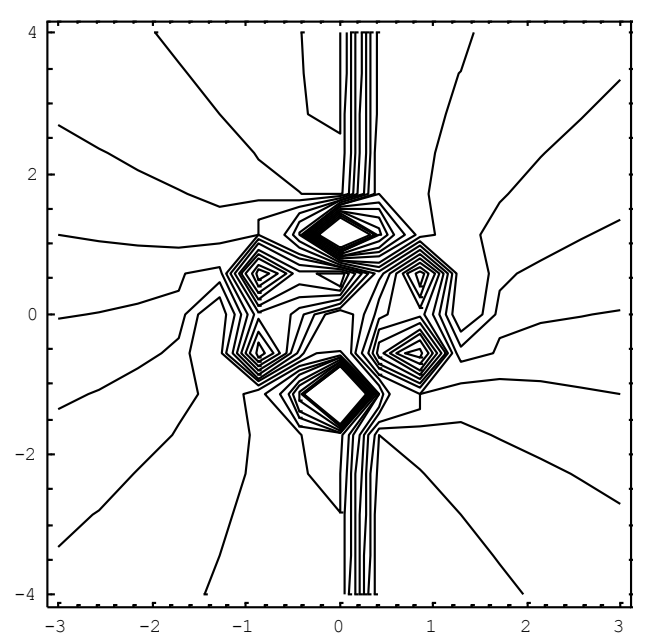

Fig. 22: Streamlines Pattern for $\operatorname{Tan}^{-1}\left(\frac{y}{x}\right)-\frac{200}{-400\left(x^{2}+y^{2}\right)+500}$

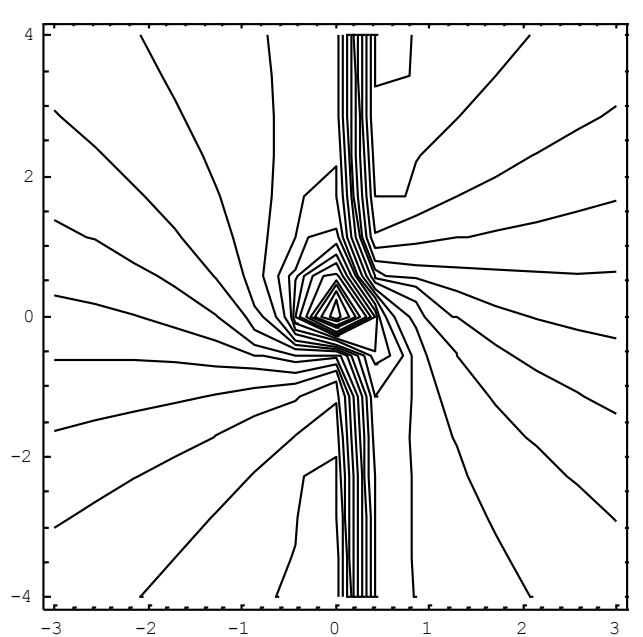

Fig. 19: Streamlines Pattern for $\operatorname{Tan}^{-1}\left(\frac{y}{x}\right)-\frac{17.8}{20\left(x^{2}+y^{2}\right)+3}$

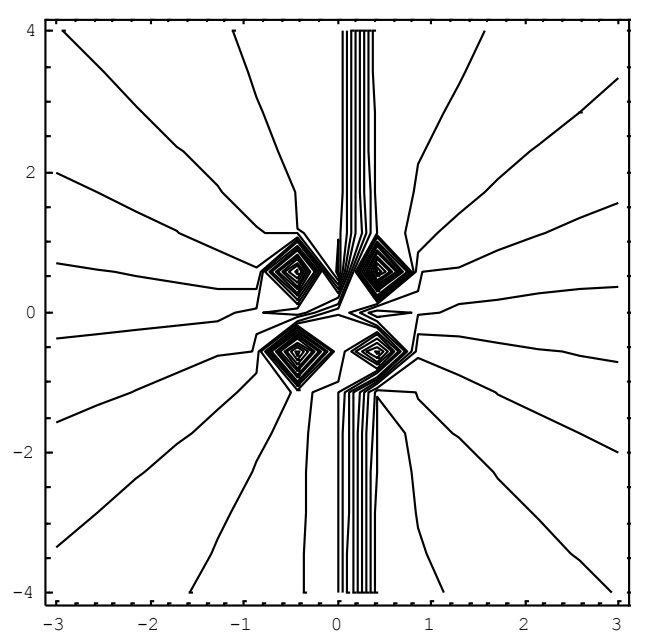

Fig. 21: Streamlines Pattern for $\operatorname{Tan}^{-1}\left(\frac{y}{x}\right)-\frac{17.8}{-400\left(x^{2}+y^{2}\right)+200}$

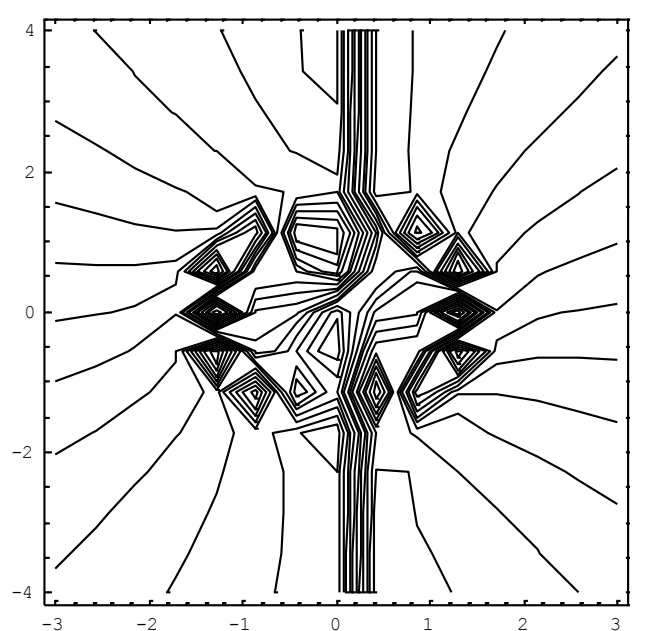

Fig. 23: Streamlines Pattern for $\operatorname{Tan}^{-1}\left(\frac{y}{x}\right)-\frac{200}{-500\left(x^{2}+y^{2}\right)+900}$ 


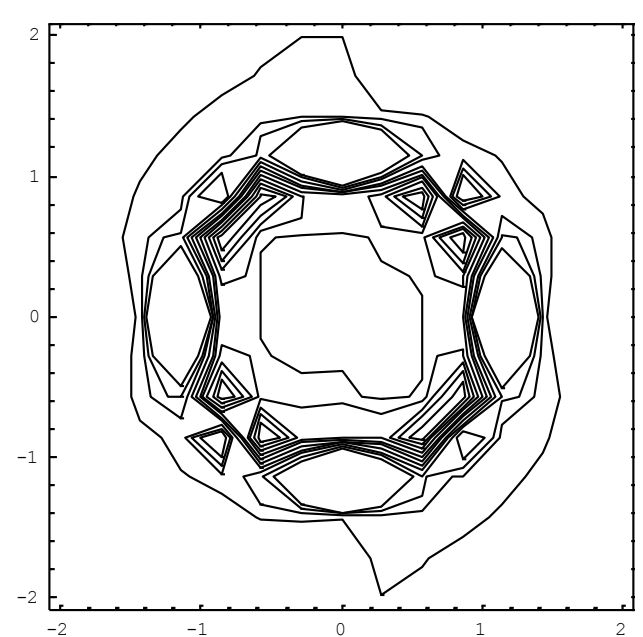

Fig. 24: Streamlines Pattern for $\operatorname{Tan}^{-1}\left(\frac{y}{x}\right)+\frac{510}{80\left(x^{2}+y^{2}\right)-100}$

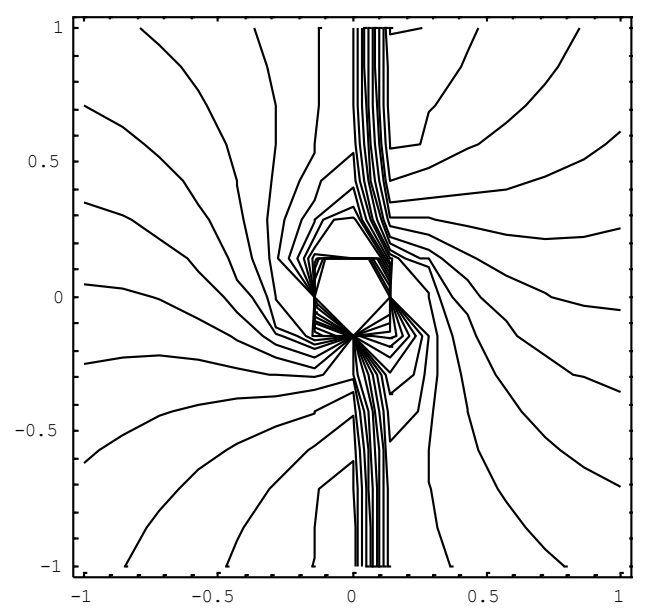

Fig. 51: Streamlines Pattern for $\operatorname{Tan}^{-1}\left(\frac{y}{x}\right)-\ln \sqrt{\left(x^{2}+y^{2}\right)}-\frac{1}{\sqrt{x^{2}+y^{2}}}$

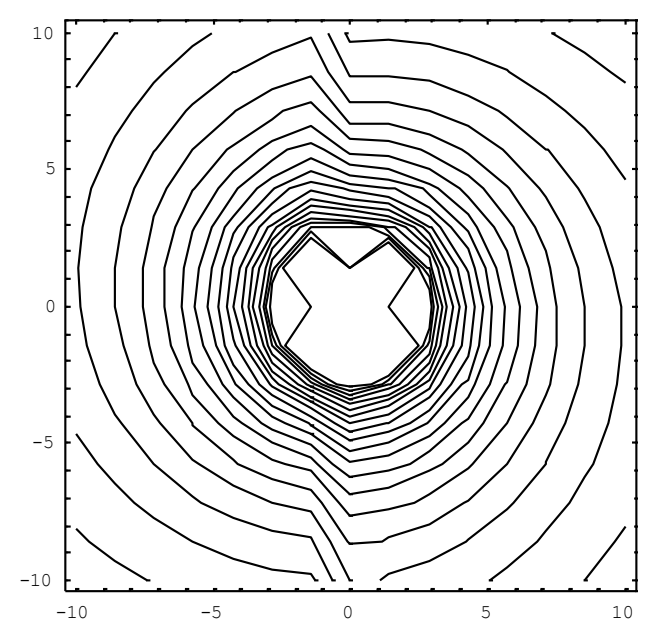

Fig. 53: Streamlines Pattern for $\operatorname{Tan}^{-1}\left(\frac{y}{x}\right)-\ln \sqrt{\left(x^{2}+y^{2}\right)}-\frac{100}{\sqrt{x^{2}+y^{2}}}$

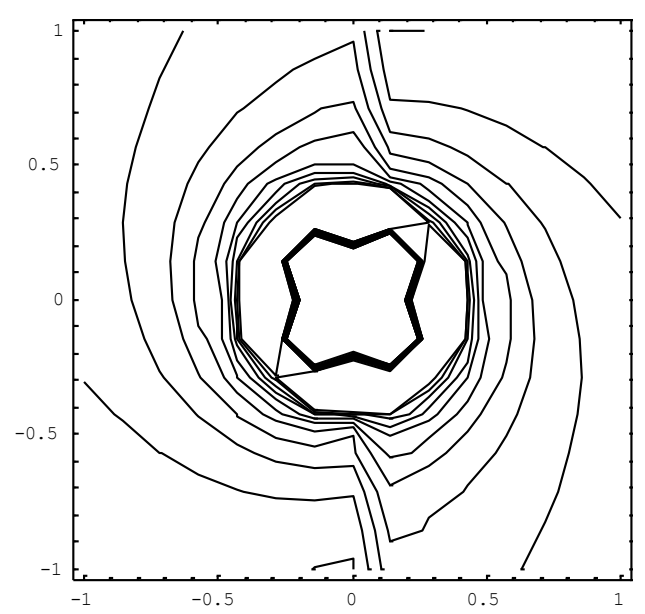

Fig. 25: Streamlines Pattern for $\operatorname{Tan}^{-1}\left(\frac{y}{x}\right)-\frac{210}{180\left(x^{2}+y^{2}\right)-10}$

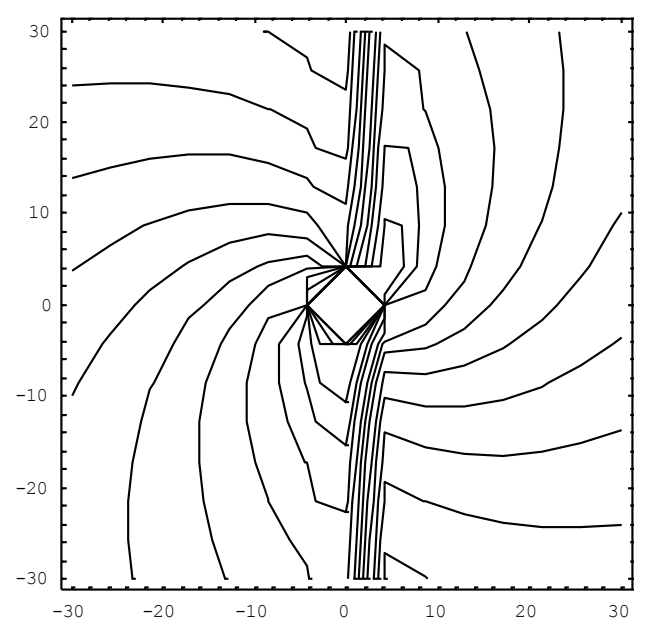

Fig. 52: Streamlines Pattern for $\operatorname{Tan}^{-1}\left(\frac{y}{x}\right)-\ln \sqrt{\left(x^{2}+y^{2}\right)}+\frac{0.69}{\sqrt{x^{2}+y^{2}}}$

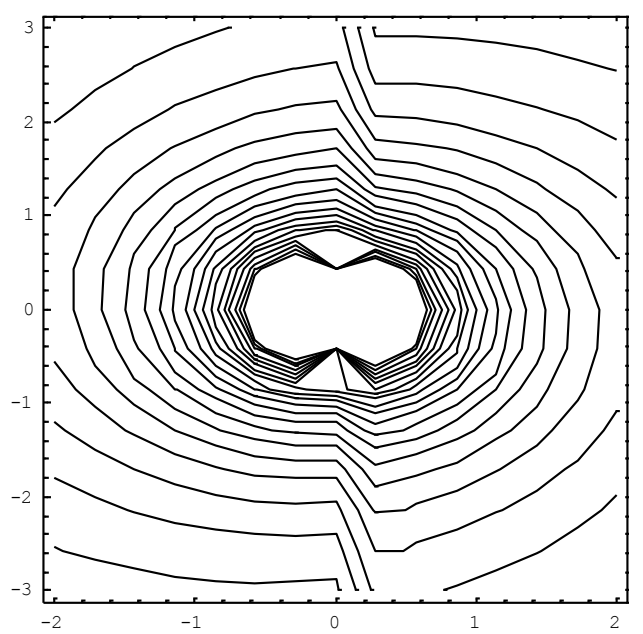

Fig. 54: Streamlines Pattern for $\operatorname{Tan}^{-1}\left(\frac{y}{x}\right)-\ln \sqrt{\left(x^{2}+y^{2}\right)}-\frac{20.1606}{\sqrt{x^{2}+y^{2}}}$ 


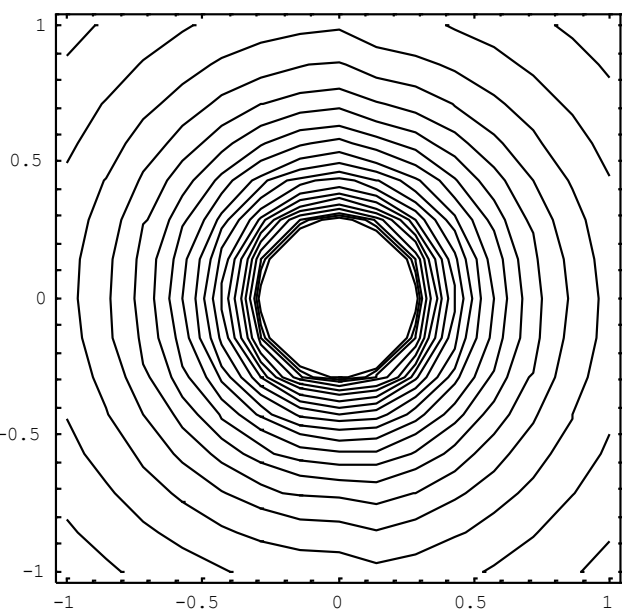

Fig. 55: Streamlines Pattern for $\operatorname{Tan}^{-1}\left(\frac{y}{x}\right)-\ln \sqrt{\left(x^{2}+y^{2}\right)}-\frac{50}{\sqrt{x^{2}+y^{2}}}$

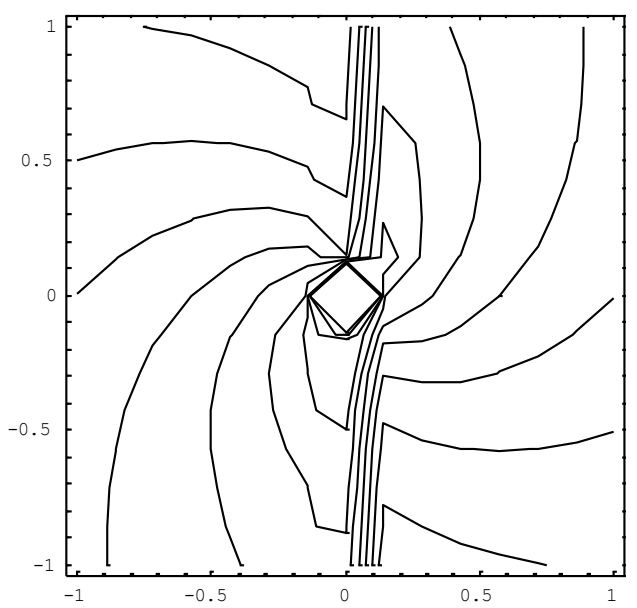

Fig. 56: Streamlines Pattern for

$\operatorname{Tan}^{-1}\left(\frac{y}{x}\right)-\ln \sqrt{\left(x^{2}+y^{2}\right)}-\frac{4.26652 \times 10^{-15}}{\sqrt{x^{2}+y^{2}}}$

\section{References}

[1] Berker R (1982), an exact solution of the Navier-Stokes equations, International Journal of Engineering and Science, 22, pp.217-230. http://dx.doi.org/10.1016/0020-7225(82)90017-9.

[2] Chandna OP and Oku-Ukpong EO (1994). Flows for chosen vorticity functions-Exact Solutions of the Navier-Stokes Equations, International Journal of Applied Mathematics and Mathematical Sciences, 17, pp.155-164. http://dx.doi.org/10.1155/S0161171294000219.

[3] Dorrepaal JM (1986), An exact solution of the Navier-Stokes equations which describes non-orthogonal stagnation -point flow in two dimensions, Journal of Fluid Mechanics, 163, pp. 141-147. http://dx.doi.org/10.1017/S0022112086002240.

[4] Hui WH (1987), Exact solutions of the unsteady two-dimensional Navier-Stokes equations, Journal of Applied Mathematics and Physics, 38, pp. 689-702 http://dx.doi.org/10.1007/BF00948290.

[5] Kovasznay LIG (1948), Laminar flow behind a two-dimensional grid, Proceedings of the Cambridge Philosophical Society, 44, pp. 58-62. http://dx.doi.org/10.1017/S0305004100023999.

[6] Kapitanskiy LV (1979), Group analysis of the Navier-Stokes equations in the presence of rotational symmetry and some new exact solutions, Zapiski nauchnogo sem, LOMI. 84, pp. 89-107.

[7] Kambe $\mathrm{T}$ (1986), A class of exact solutions of the Navier-Stokes equations, Fluid Dynamics Research, 1, pp.2131http://dx.doi.org/10.1016/0169-5983(86)90004-3.

[8] Martin M H (1971), the flow of a viscous fluid I, Archive for Rational Mechanics and Analysis, 41(4), pp.266-286. http://dx.doi.org/10.1007/BF00250530.

[9] Rana Khalid Naeem (1994), Exact solutions of flow equations of an incompressible fluid of variable viscosity via one - parameter group, The Arabian Journal for Science and Engineering, 19, pp.111-114.

[10] Rana Khalid Naeem and Syed Anwer Ali (1996), Exact solutions of the equations of motion of an incompressible fluid of variable viscosity, Karachi University Journal of Science, 24, pp. 35-40.

[11] Rana Khalid Naeem and Sarfaraz Ahmed Nadeem (1996), Study of steady plane flows of an incompressible fluid of variable viscosity using Martin's System, Journal of Applied Mechanics and Engineering, 1, pp.397-433.

[12] Rana Khalid Naeem and Syed Anwer Ali (2001), A class of exact solutions to equations governing the steady plane flows of an incompressible fluid of variable viscosity via von-Mises variables, International Journal of Applied Mechanics and Engineering, 6, pp.395436.

[13] Rana Khalid Naeem (2003), Steady plane flows of an incompressible fluid of variable viscosity via Hodograph transformation method, Karachi University Journal of Sciences, 3, pp. 73-89.

[14] Rana Khalid Naeem and Muhammad Jamil (2005), A class of exact solutions to flow equations of an incompressible fluid of variable viscosity, Quaid-e-Awam University Research Journal of Engineering Science and Technology, 6(1, 2), pp. 11-18.

[15] Rana Khalid Naeem and Muhammad Jamil (2006), on plane steady flows of an incompressible fluid with variable viscosity, International Journal of Applied Mathematics and Mechanics, 2(3), pp. 32-51.

[16] Rana Khalid Naeem (2007), on plane flows of an incompressible fluid of variable viscosity, Quarterly Science Vision, 12, pp.125-131.

[17] Rana Khalid Naeem and Sobia Younus (2010), exact solutions of the Navier-Stokes equations for incompressible fluid of variable viscosity for prescribed vorticity distributions, International Journal of Applied Mathematics and Mechanics, 6(5), pp. 18-38.

[18] Rana Khalid Naeem and Sobia Younus (2014), A class of exact solutions of the Navier-Stokes equations for incompressible fluid of variable viscosity, International Journal of Applied Mathematics and Mechanics, 10(2), pp. 93-121

[19] Wang C Y(1966), on a class of exact solutions of the Navier-Stocks equations, Journal of Applied Mechanics, 33, pp.696-698 http://dx.doi.org/10.1115/1.3625151.

[20] Wang C Y (1989), Exact solutions of the unsteady Navier-Stokes Equations, Applied Mechanics Review, 42, pp.269-282. http://dx.doi.org/10.1115/1.3152400.

[21] Wang C Y (1990). Exact solutions of the Navier- Stokes equations-the generalized Beltrami flows, review and extension, Acta Mechanica, 81, pp.69-74. http://dx.doi.org/10.1007/BF01174556.

[22] Wang C Y (1991), Exact solutions of the steady-state Navier-Stocks equations, Annual Review, Fluid Mechanics, 23, pp.159-177. http://dx.doi.org/10.1146/annurev.fl.23.010191.001111.

[23] Wang C Y (2003), Stagnation flows with slip: exact solutions of the Navier-Stokes equations, Zeitschrift fur Angewandte Mathematik und Physik, 54(1), pp.184-189.http://dx.doi.org/10.1007/PL00012632. 